\title{
Efficient Syntheses of Rugulosin Analogues
}

Barry B. Snider* and Xiaolei Gao

Department of Chemistry MS 015, Brandeis University, Waltham, MA 02454-9110

snider@brandeis.edu

\section{Supporting Material}

Preparation of $\mathbf{1 2}$

S2

Table of ${ }^{13} \mathrm{C}$ NMR Spectral Data of 2b, 20, 10b, 18, 21, and 23

S5

Copies of ${ }^{1} \mathrm{H}$ and ${ }^{13} \mathrm{C}$ NMR spectra

S6-S37 
2-Methoxy-4-methylbenzoic acid (24). To a solution of 4-methylsalicylic acid (3.00 g, 19.74 mmol) in acetone $(60 \mathrm{~mL})$ was added $\mathrm{K}_{2} \mathrm{CO}_{3}(10.90 \mathrm{~g}, 78.92 \mathrm{mmol})$ and $\mathrm{MeI}(12.30 \mathrm{~mL}, 197.4$ mmol). The mixture was heated at $65^{\circ} \mathrm{C}$ for $12 \mathrm{~h}$ and cooled to $25^{\circ} \mathrm{C}$. The resulting reaction mixture was filtered and the residue was washed with acetone. The filtrate and acetone wash were combined and concentrated to give $4 \mathrm{~g}$ of crude methyl 2-methoxy-4-methylbenzoate, ${ }^{18}$ which was dissolved in $60 \mathrm{~mL}$ of $1 \mathrm{M}$ aqueous $\mathrm{NaOH}$ solution. The basic solution was heated to reflux for $16 \mathrm{~h}$ and cooled to $0{ }^{\circ} \mathrm{C}$. Concentrated $\mathrm{HCl}$ was added to precipitate 24 . The white solid was filtered and dried in vacuo to give $3.2 \mathrm{~g}$ (98\%) of 24 with ${ }^{1} \mathrm{H}$ NMR spectral data identical to those previously reported. ${ }^{19}$

N,N-Diethyl-2-methxoy-4-methylbenzamide (25). Thionyl chloride (21 mL, $289.1 \mathrm{mmol}$ ) was added to 24 (3.2 g, $19.28 \mathrm{mmol}$ ) at $25^{\circ} \mathrm{C}$ with stirring, resulting in slow effervescence. To this reaction mixture was added DMF $(50 \mu \mathrm{L})$ dropwise via a syringe after which effervescence became more vigorous. The reaction mixture was stirred at $25^{\circ} \mathrm{C}$ for $30 \mathrm{~min}$, at which time no further effervescence was visible. Half of the thionyl chloride was removed under reduced pressure, toluene $(10 \mathrm{~mL})$ was added and the mixture was evaporated to dryness. A further 10 $\mathrm{mL}$ of toluene was added and the process of evaporation was repeated twice more to ensure complete removal of all excess thionyl chloride. The pale yellow oil was dissolved in dry THF (20 mL), cooled to $0{ }^{\circ} \mathrm{C}$ and treated slowly with diethylamine $(7.6 \mathrm{~mL}, 73.26 \mathrm{mmol})$ with stirring to prevent the reaction from becoming too vigorous. The reaction mixture was stirred for a further 10 min and diluted with $\mathrm{CH}_{2} \mathrm{Cl}_{2}$, washed with $\mathrm{H}_{2} \mathrm{O}$ and brine, dried $\left(\mathrm{MgSO}_{4}\right)$, and concentrated to give 25 (4.2 g, 99\%) as a straw-colored liquid that solidified at $25{ }^{\circ} \mathrm{C}$. The ${ }^{1} \mathrm{H}$ NMR spectral data of $\mathbf{2 5}$ are identical to those previously reported. ${ }^{20}$ 
N,N-Diethyl-3-formyl-2-methxoy-4-methylbenzamide (26). To a stirred solution of dry THF (70 mL) at $-78{ }^{\circ} \mathrm{C}$ was added TMEDA (3.73 mL, $\left.24.70 \mathrm{mmol}\right)$ followed by s-BuLi (1.4 M in cyclohexane, $17.64 \mathrm{~mL}, 24.70 \mathrm{mmol}){ }^{21}$ Amide 25 (4.2 g, $\left.19.00 \mathrm{mmol}\right)$ in dry THF (15 mL) was added to the reaction mixture dropwise via a cannula over $30 \mathrm{~min}$. The resulting reaction mixture was stirred at $-78^{\circ} \mathrm{C}$ for another $30 \mathrm{~min}$ and DMF (1.77 mL, $\left.22.80 \mathrm{mmol}\right)$ was added dropwise. The cooling bath was removed after $5 \mathrm{~min}$ and the reaction mixture was allowed to warm to $25{ }^{\circ} \mathrm{C}$ and stirred for an additional $16 \mathrm{~h}$. The reaction mixture was cooled to $0{ }^{\circ} \mathrm{C}$ and acidified to $\mathrm{pH} 4-5$ by addition of $6 \mathrm{M}$ aqueous $\mathrm{HCl}$ solution. The organic layer was separated and the aqueous layer was extracted with EtOAc $(3 \times 80 \mathrm{~mL})$. The combined organic layers were washed with $\mathrm{H}_{2} \mathrm{O}$, brine, dried $\left(\mathrm{Na}_{2} \mathrm{SO}_{4}\right)$, and concentrated to give $\mathbf{2 6}$ (4.7 g, 99\%) as a pale yellow oil: ${ }^{1} \mathrm{H}$ NMR 9.97 (s, 1), 7.35 (s, 1), 6.97 (s, 1), 3.85 (s, 3), 3.73 (1, dq, J= 13.4, 6.7), 3.53 (1, dq, $J=13.4,6.7), 3.11$ (q, 2, $J=6.7), 2.43$ (s, 3), 1.29 (dd, 3, $J=6.7,6.7), 1.01$ (t, 3, $J=$ 6.7).

3-Hydroxy-7-methoxy-5-methyl-1(3H)-isobenzofuranone (27). A solution of 26 (4.7 g, $18.88 \mathrm{mmol}$ ) in $40 \mathrm{~mL}$ of $3 \mathrm{M}$ aqueous $\mathrm{HCl}$ solution and $40 \mathrm{~mL}$ of $\mathrm{AcOH}$ was stirred at reflux for $20 \mathrm{~h}$ and cooled to $25^{\circ} \mathrm{C}$. Removal of the solvent under vacuum gave a brown residue that was extracted with EtOAc $(3 \times 20 \mathrm{~mL})$. The combined EtOAc layers were extracted with saturated aqueous $\mathrm{NaHCO}_{3}$ solution $(3 \times 20 \mathrm{~mL})$. The combined base phase was acidified by concentrated $\mathrm{HCl}$ forming a white precipitate. Removal of the solvent by filtration gave 27 (3.0 g, 82\%) as a white solid with ${ }^{1} \mathrm{H}$ NMR spectral data identical to those previously reported. ${ }^{10 c}$

1,3-Dihydro-4-methoxy-6-methyl-3-oxo-1-isobenzofurancarbonitrile (12). To a stirred solution of KCN (4.83 g, $74.23 \mathrm{mmol})$ in water (30 mL) was added 27 (3.0 g, $15.46 \mathrm{mmol})$. The clear yellow solution was stirred at $25^{\circ} \mathrm{C}$ for $10 \mathrm{~min}$ and cooled to $0{ }^{\circ} \mathrm{C}$. Ice (18 g) and 
concentrated $\mathrm{HCl}(18 \mathrm{~mL})$ were added and the resulting mixture was stored at $25^{\circ} \mathrm{C}$ for $12 \mathrm{~h}$ with the precipitation of a white solid. After filtration and drying, the white solid was purified by flash chromatography on silica gel (2:1 hexanes/EtOAc) to give 12 (2.76 g, 88\%) as a white solid with ${ }^{1} \mathrm{H}$ NMR spectral data identical to those previously reported. ${ }^{10 \mathrm{c}}$

\section{References and Notes}

(18) de Frutos, Ó.; Atienza, C.; Echavarren, A. M. Eur. J. Org. Chem. 2001, 163-171.

(19) Cass, M. E.; Garrett, T. M.; Raymond, K. N. J. Am. Chem. Soc. 1989, 111, 1677-1682.

(20) Iwao, M.; Kuraishi, T. Bull. Chem. Soc. Jpn. 1987, 60, 4051-4060.

(21) Wang, X.; de Silva, S. O.; Reed, J. N.; Billadeau, R.; Griffen, E. J.; Chan, A.; Snieckus, V. Org. Syn. 1995, 72, 163-172. 

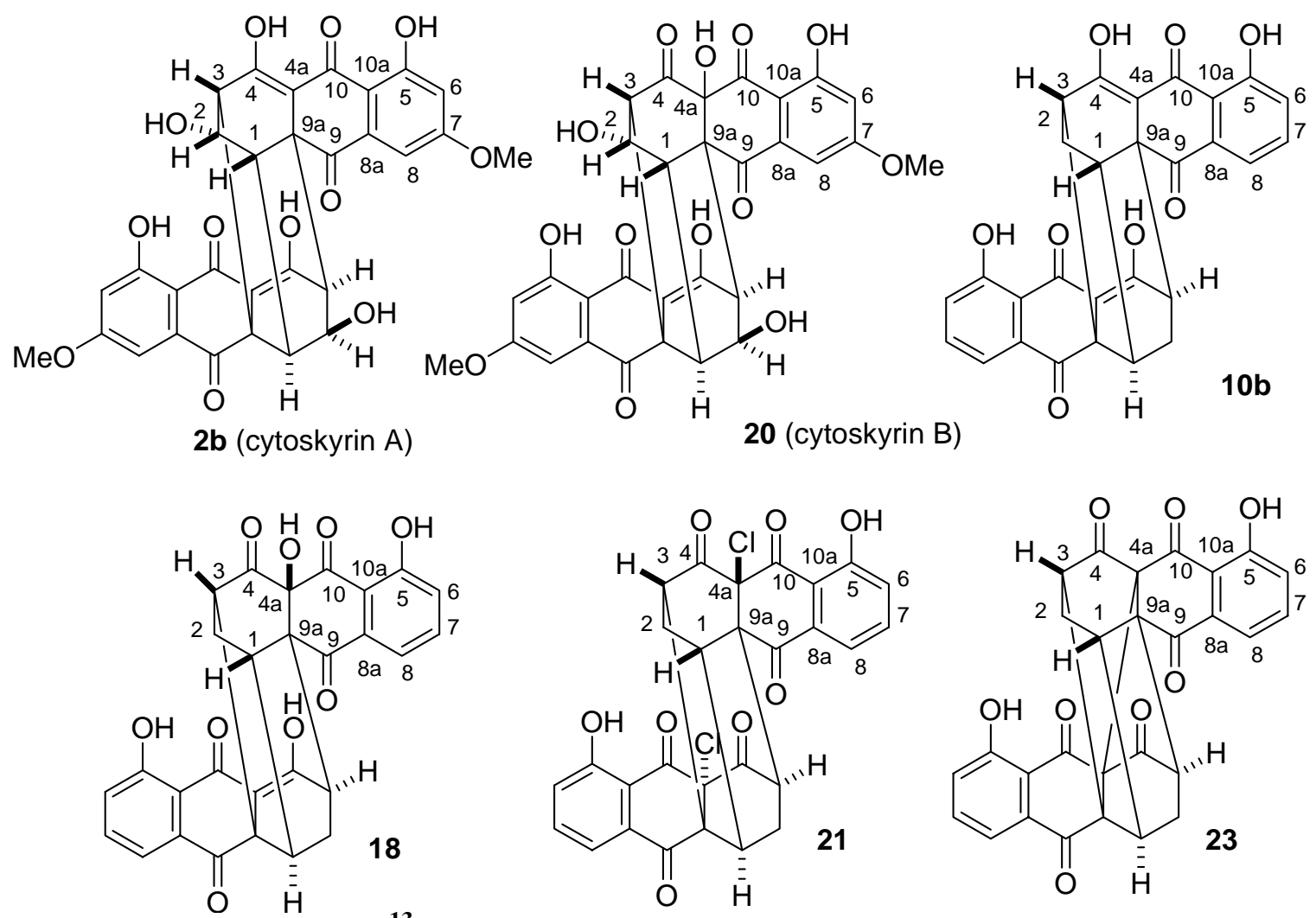

Table 1. Comparison of ${ }^{13} \mathrm{C}$ NMR Spectra Data

\begin{tabular}{|c|c|c|c|c|c|c|}
\hline Carbon $^{\mathrm{a}}$ & $\begin{array}{l}2 \mathbf{b}^{\mathrm{a}} \\
\text { both }\end{array}$ & $\begin{array}{l}\mathbf{2 0}^{\mathrm{a}} \\
\text { oxidized, unox }\end{array}$ & $\begin{array}{l}\text { 10b } \\
\text { both }\end{array}$ & $\begin{array}{l}18 \\
\text { oxidized, unox }\end{array}$ & $\begin{array}{l}21 \\
\text { both }\end{array}$ & $\begin{array}{l}23 \\
\text { both }\end{array}$ \\
\hline 1 & 51.8 & $51.5,51.2$ & 48.0 & $47.7^{*}, 44.6^{*}$ & 44.9 & 50.1 \\
\hline 2 & 74.8 & $71.2,77.7$ & 29.9 & $26.0,34.0$ & 29.7 & 38.5 \\
\hline 3 & 61.9 & $68.2,59.6$ & 54.9 & 59.9, 51.3 & 57.7 & 57.4 \\
\hline 4 & 183.5 & 199.7, 176.9 & 186.5 & $199.9,183.1$ & 187.7 & 190.6 \\
\hline $4 a$ & 106.2 & $77.0,106.8$ & 105.3 & $75.2,106.1$ & 62.5 & 75.0 \\
\hline 5 & 165.4 & $165.8,166.7$ & 161.6 & $160.7,160.9$ & 163.3 & 162.3 \\
\hline 6 & 107.1 & $107.1,106.6$ & 119.7 & $119.2,118.6$ & 120.1 & 118.8 \\
\hline 7 & 167.6 & $168.2,167.7$ & 132.2 & $133.8,132.7$ & 131.7 & 134.3 \\
\hline 8 & 108.0 & $108.2 * 108.0 *$ & 124.5 & $123.8, * 124.1^{*}$ & 125.8 & 125.0 \\
\hline $8 a$ & 137.1 & $138.6, * 138.1^{*}$ & 136.2 & $136.9, * 136.5^{*}$ & 137.3 & 137.5 \\
\hline 9 & 194.4 & $194.3,192.6$ & 194.4 & $194.1, * 193.1 *$ & 195.4 & 199.9 \\
\hline $9 a$ & 61.0 & $59.1,66.6$ & 58.6 & $56.4,63.8$ & 61.7 & 65.3 \\
\hline 10 & 188.5 & 195.6, 189.7 & 188.0 & $193.4, * 186.0$ & 189.5 & 192.0 \\
\hline 10a & 111.7 & $112.4,110.7$ & 116.6 & $116.7,115.7$ & 113.7 & 118.4 \\
\hline
\end{tabular}

OMe $\quad 56.7 \quad 57.0,57.0$

a) Data for $\mathbf{2 b}$ and $\mathbf{2 0}$ and numbering scheme taken from reference 4. *) Assignments may be switched. 

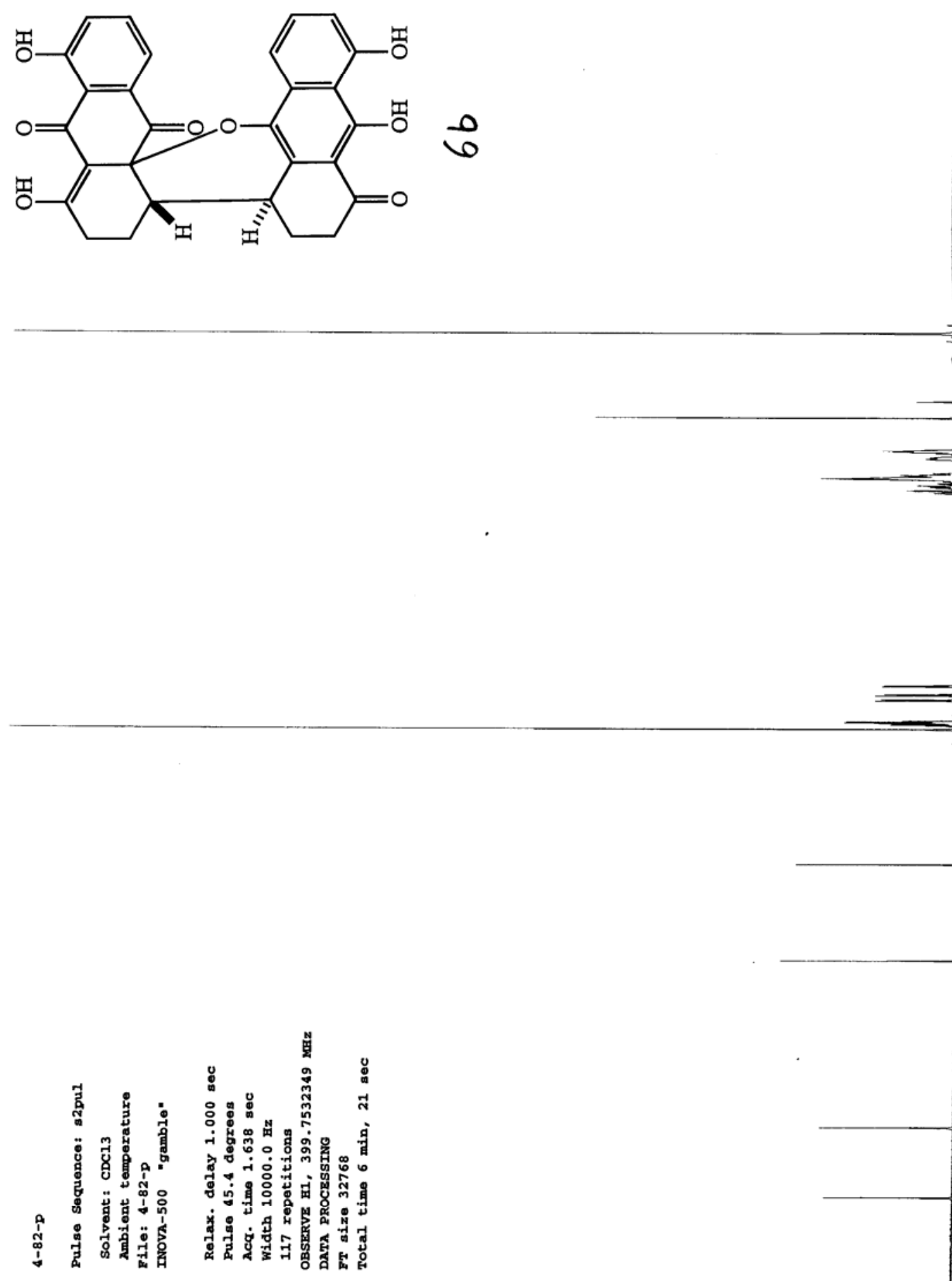

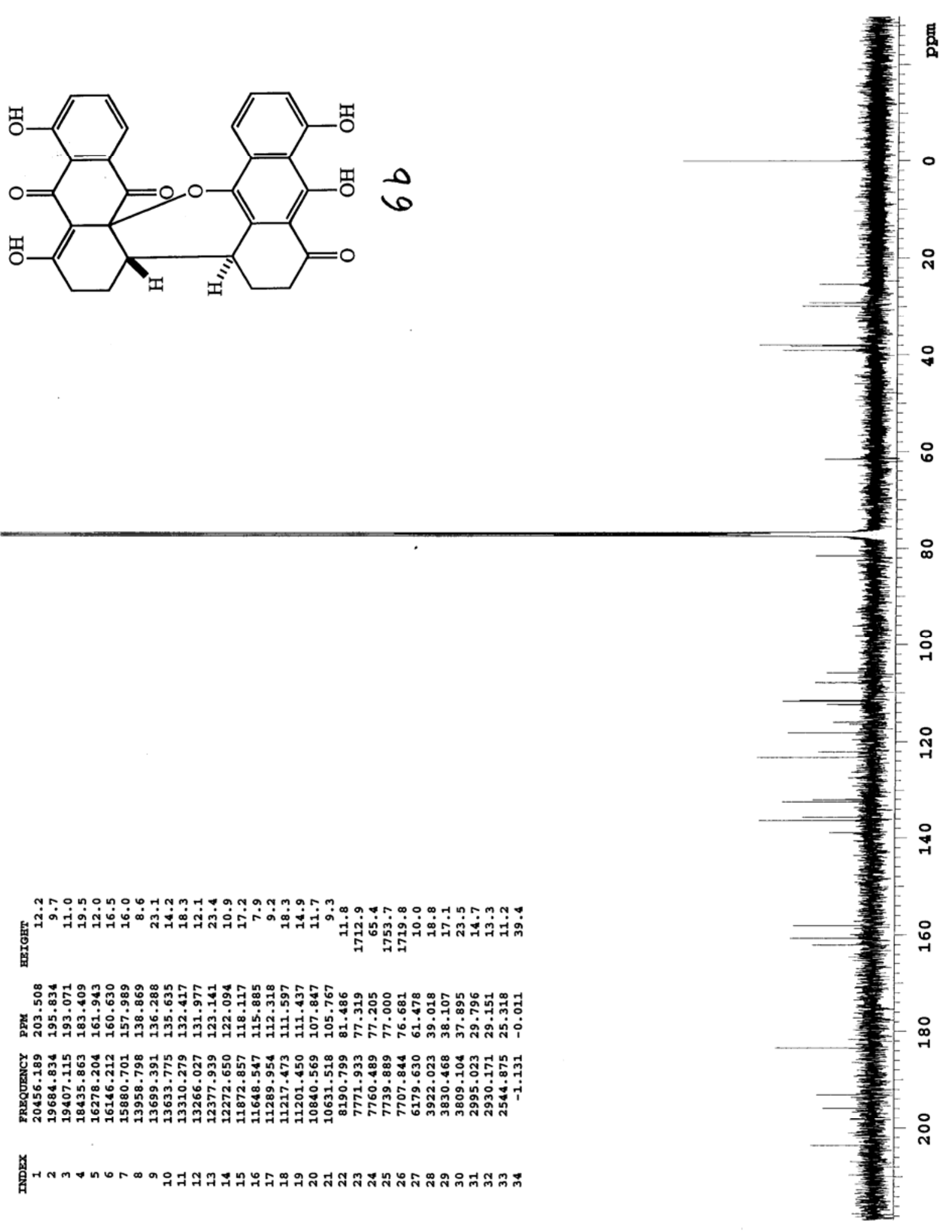

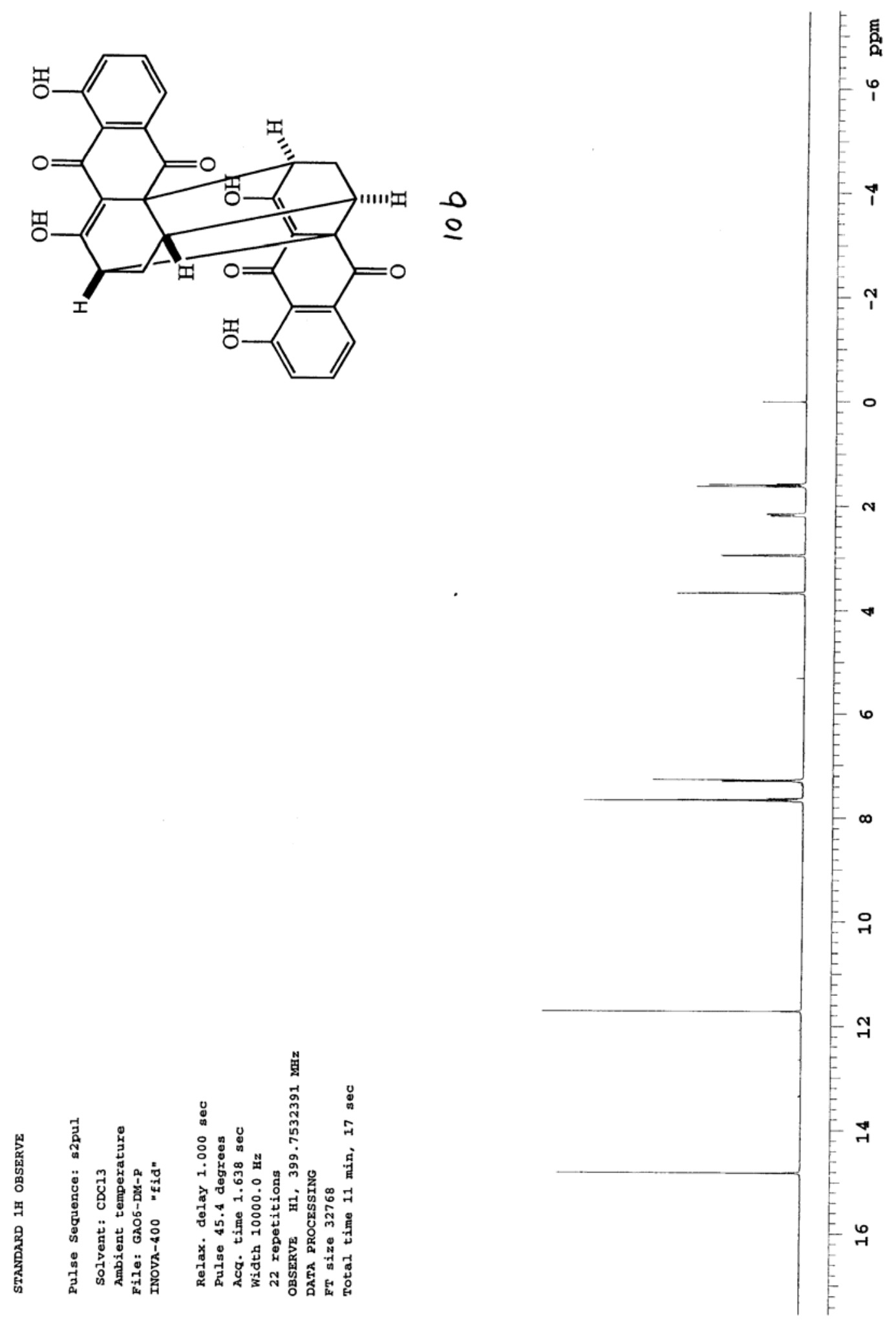


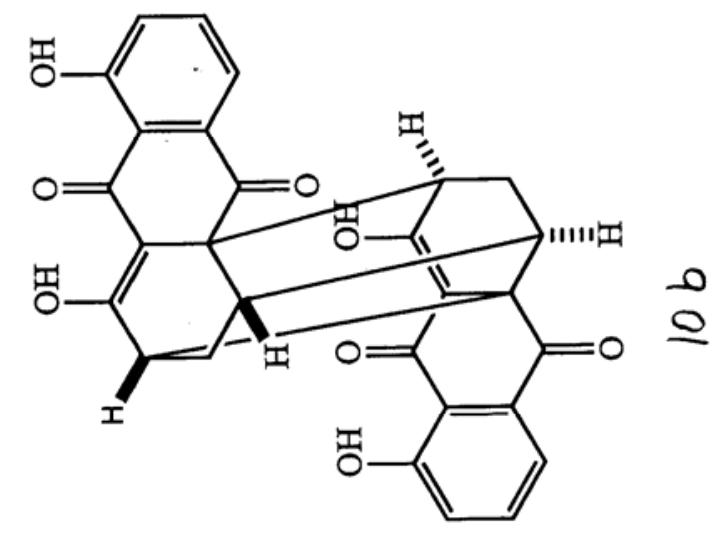

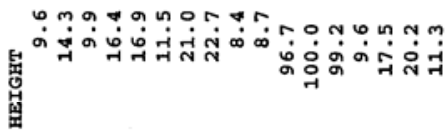

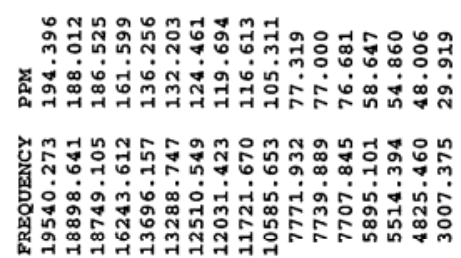

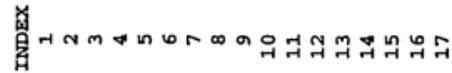

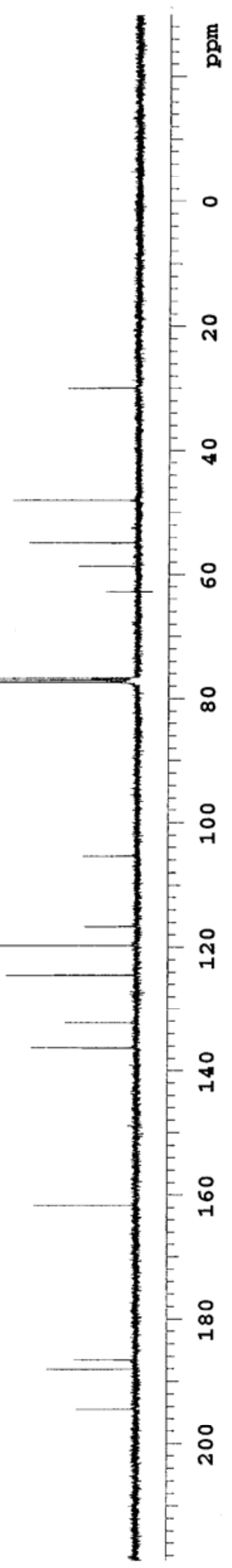




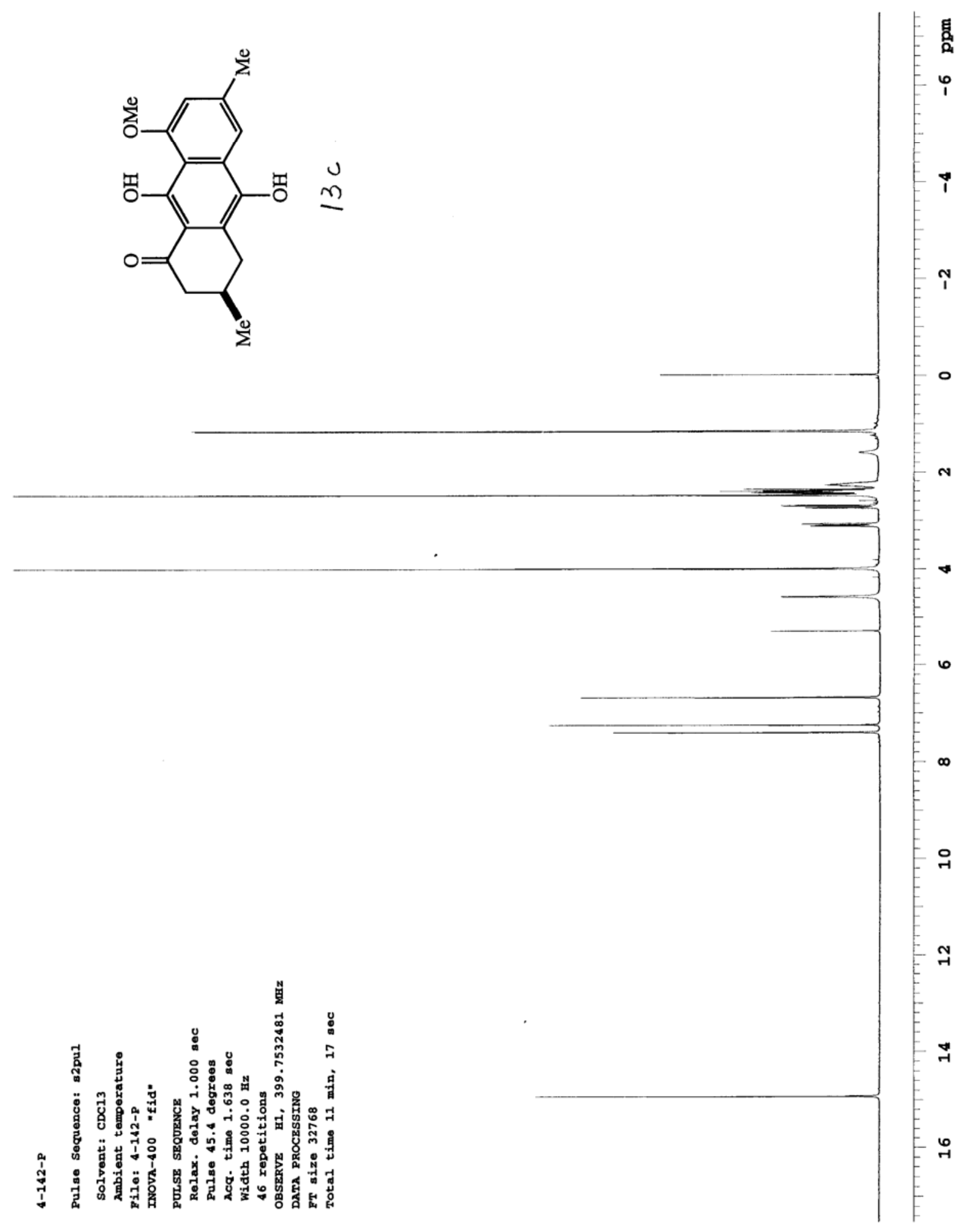



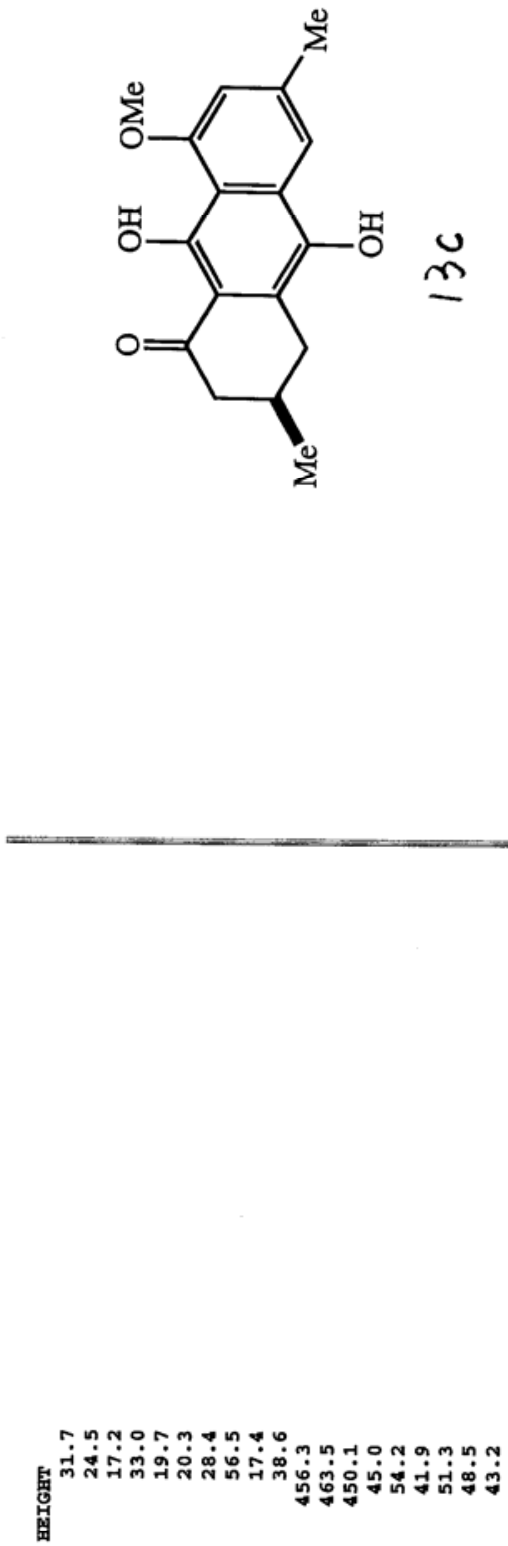

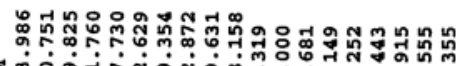

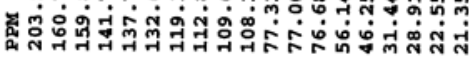

论

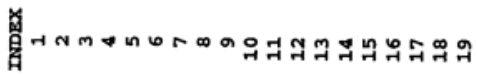

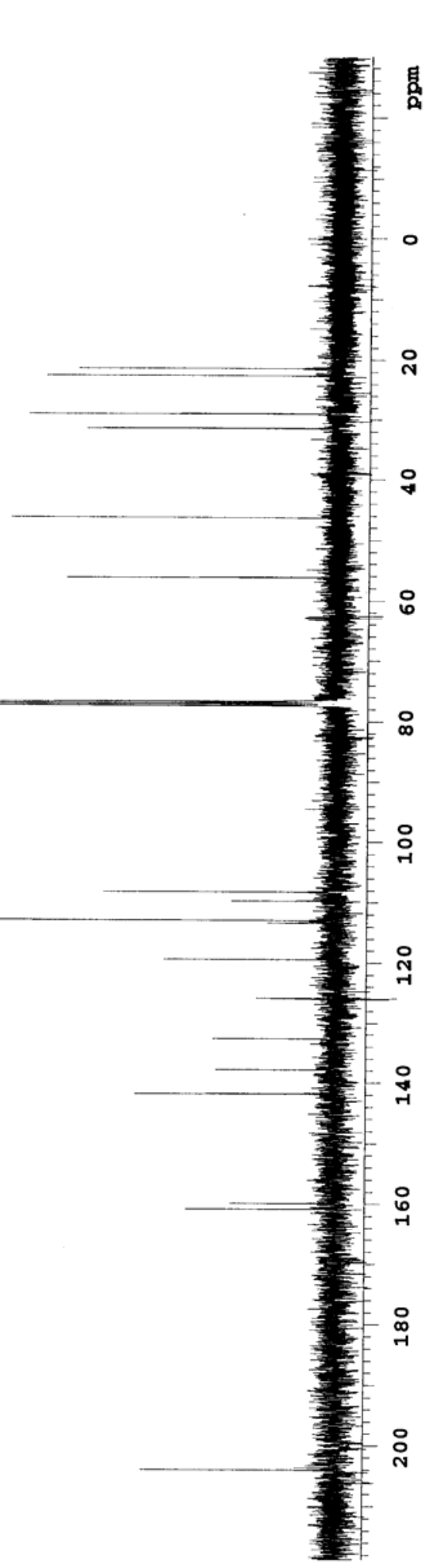



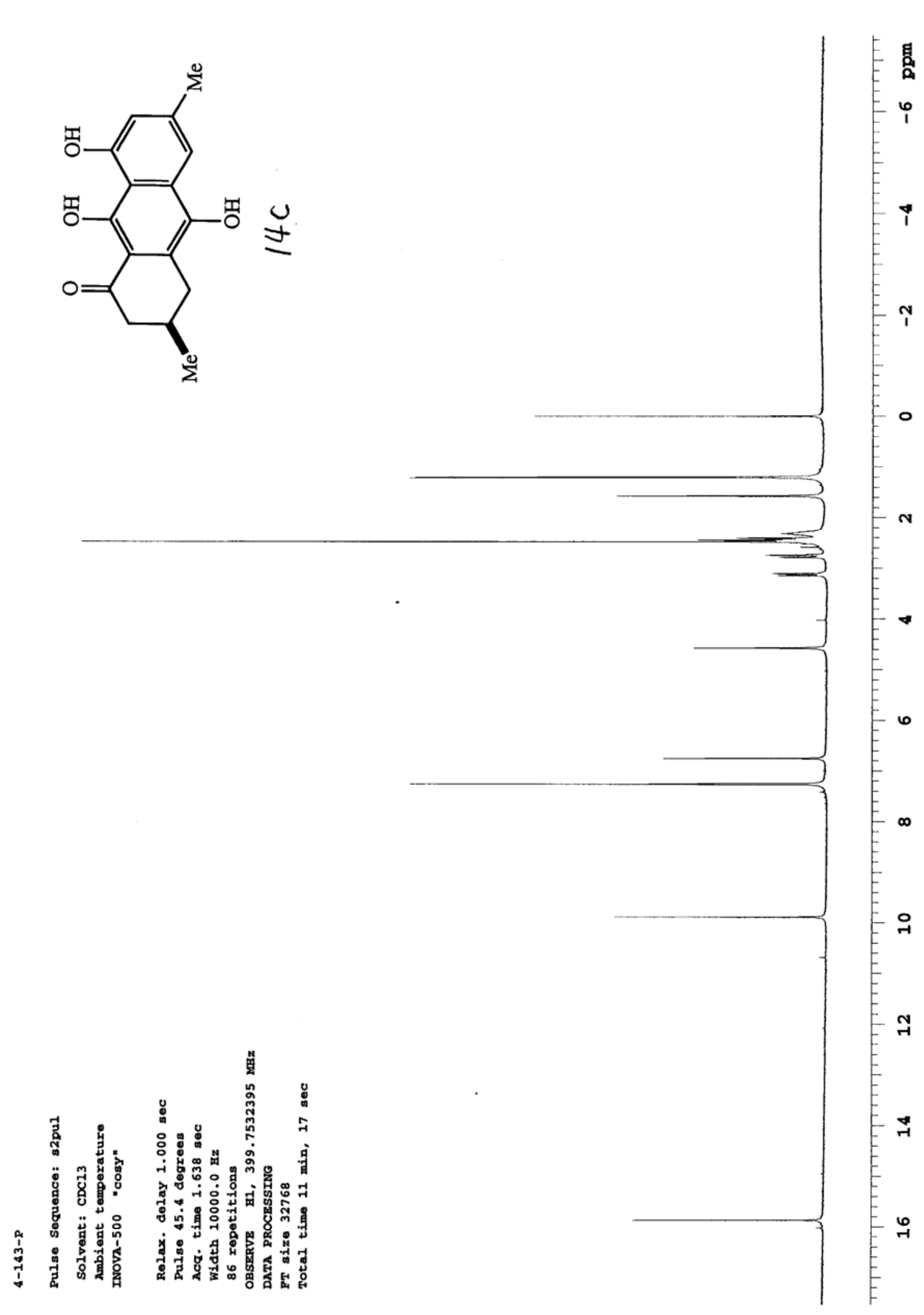


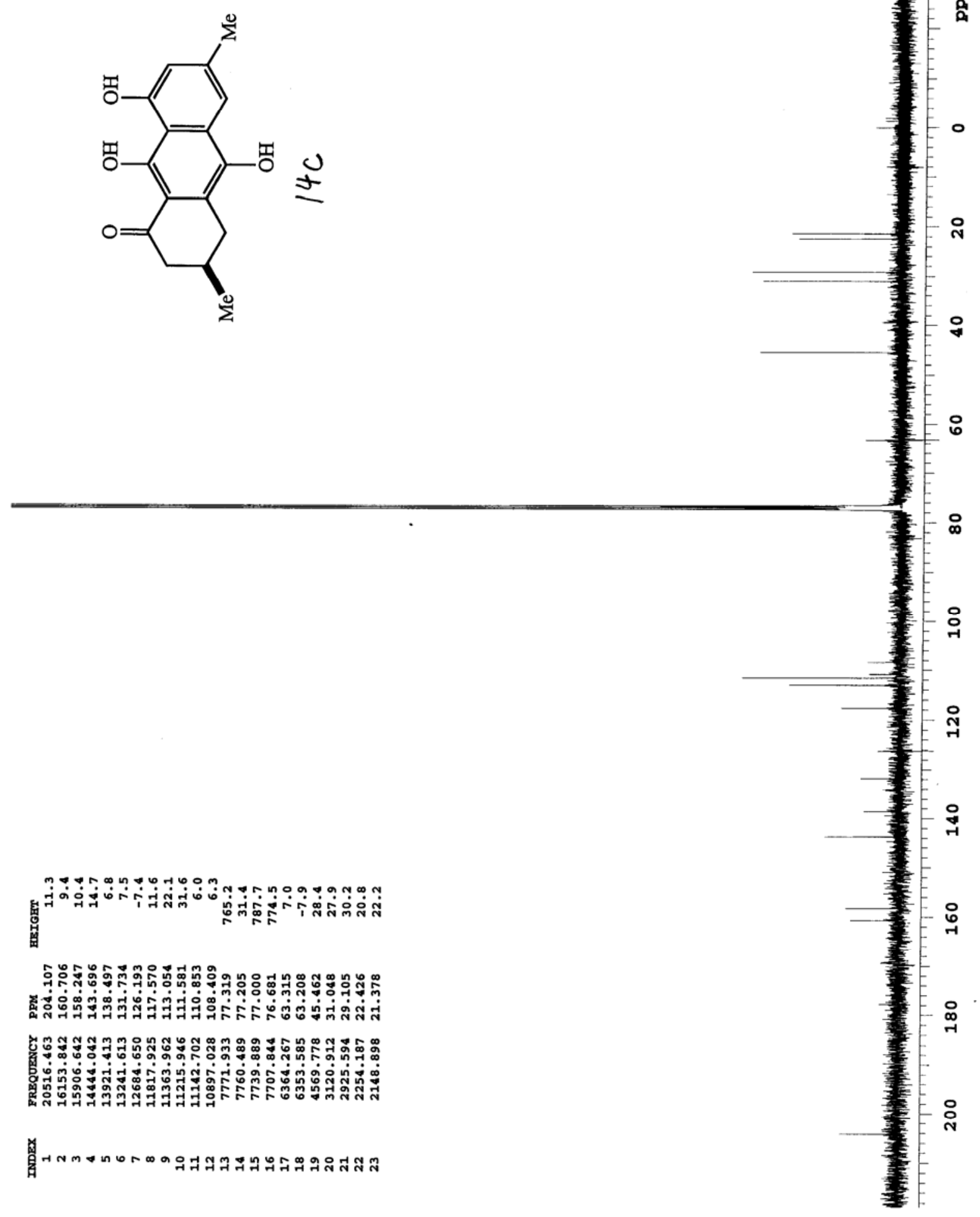




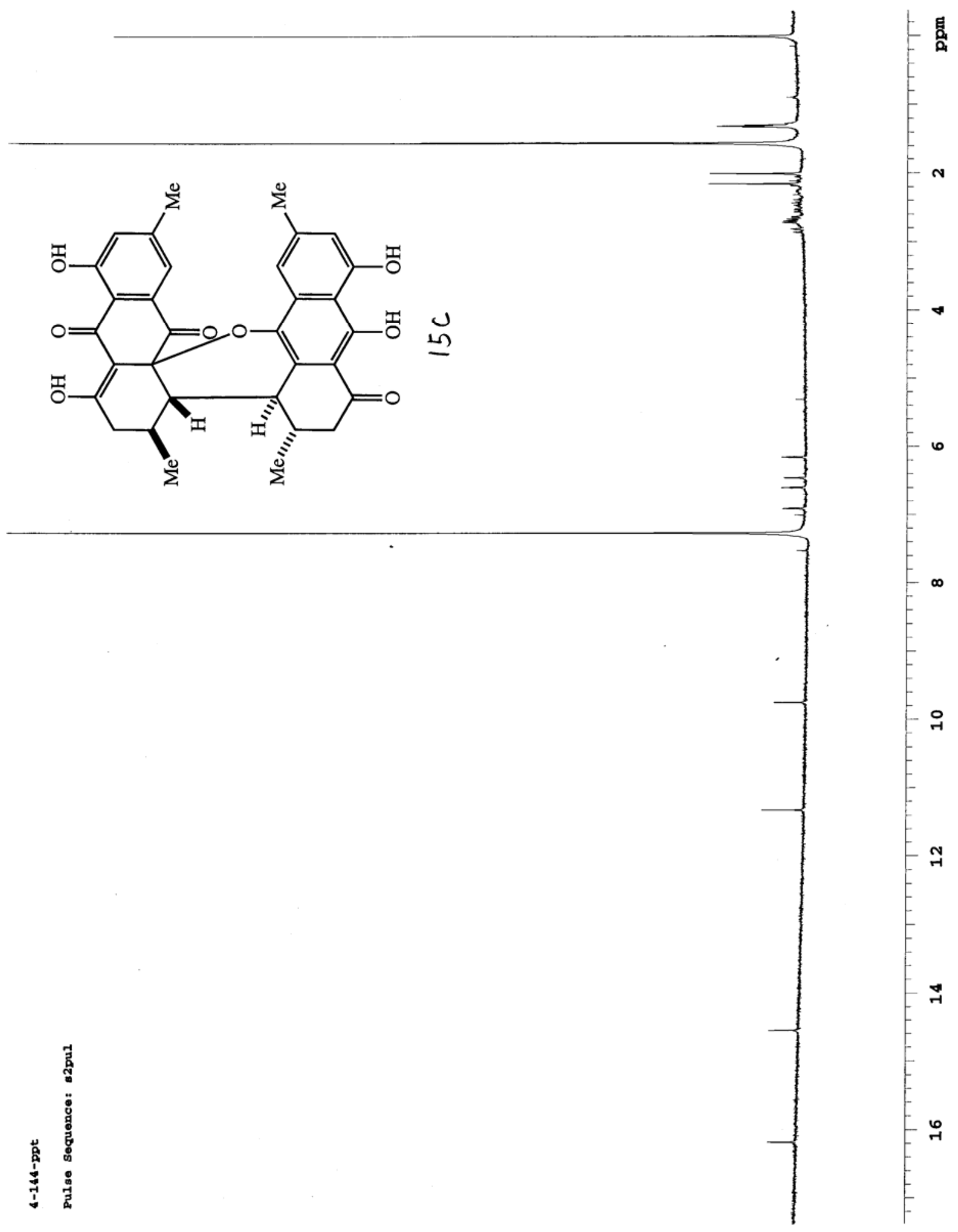




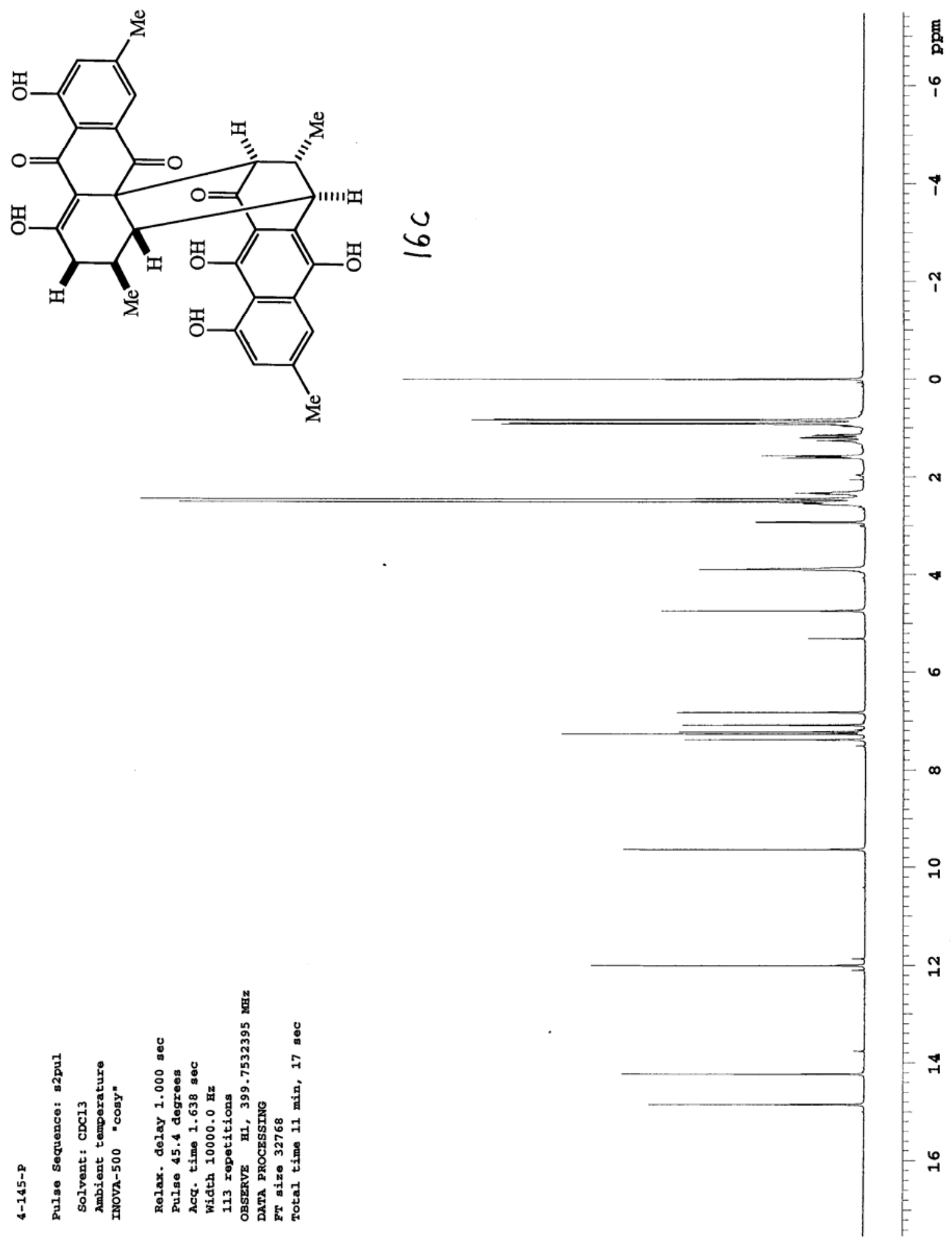



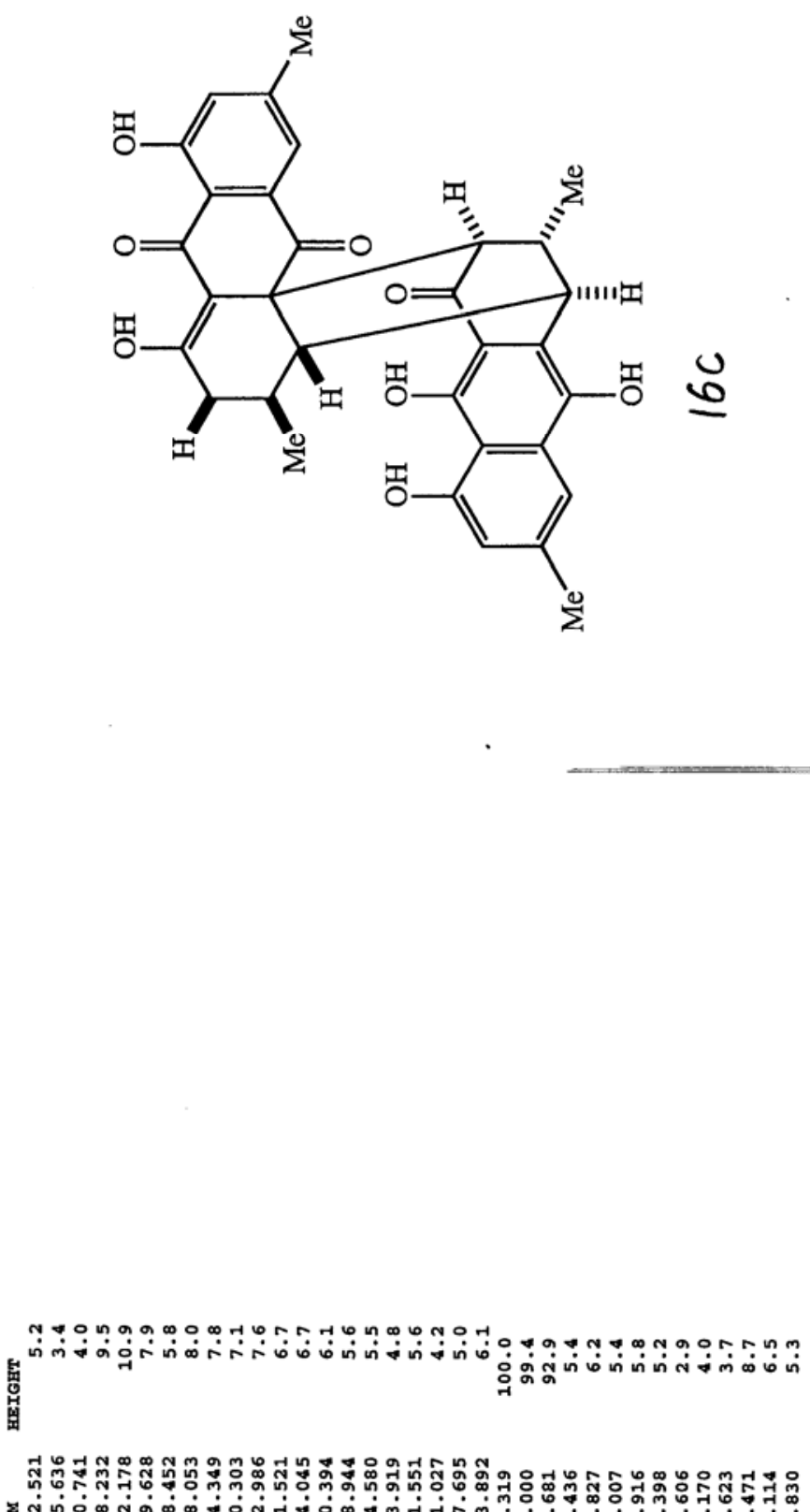
范

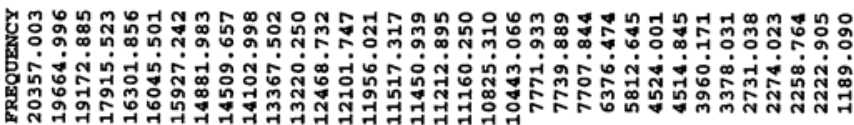

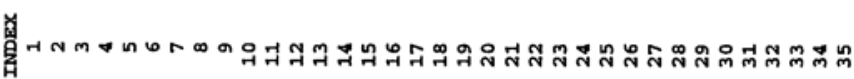

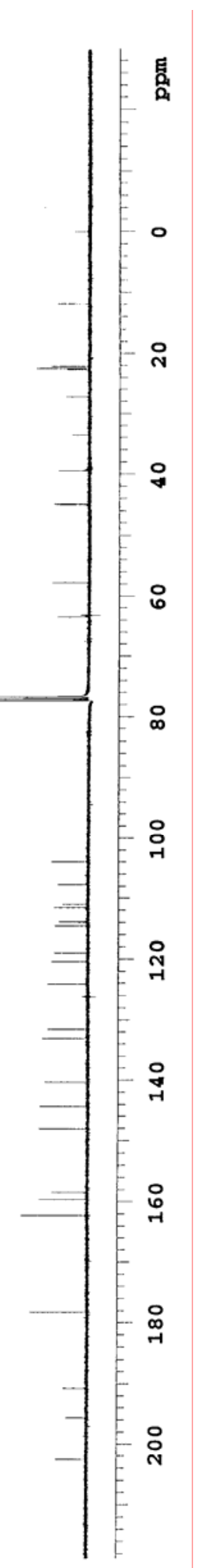



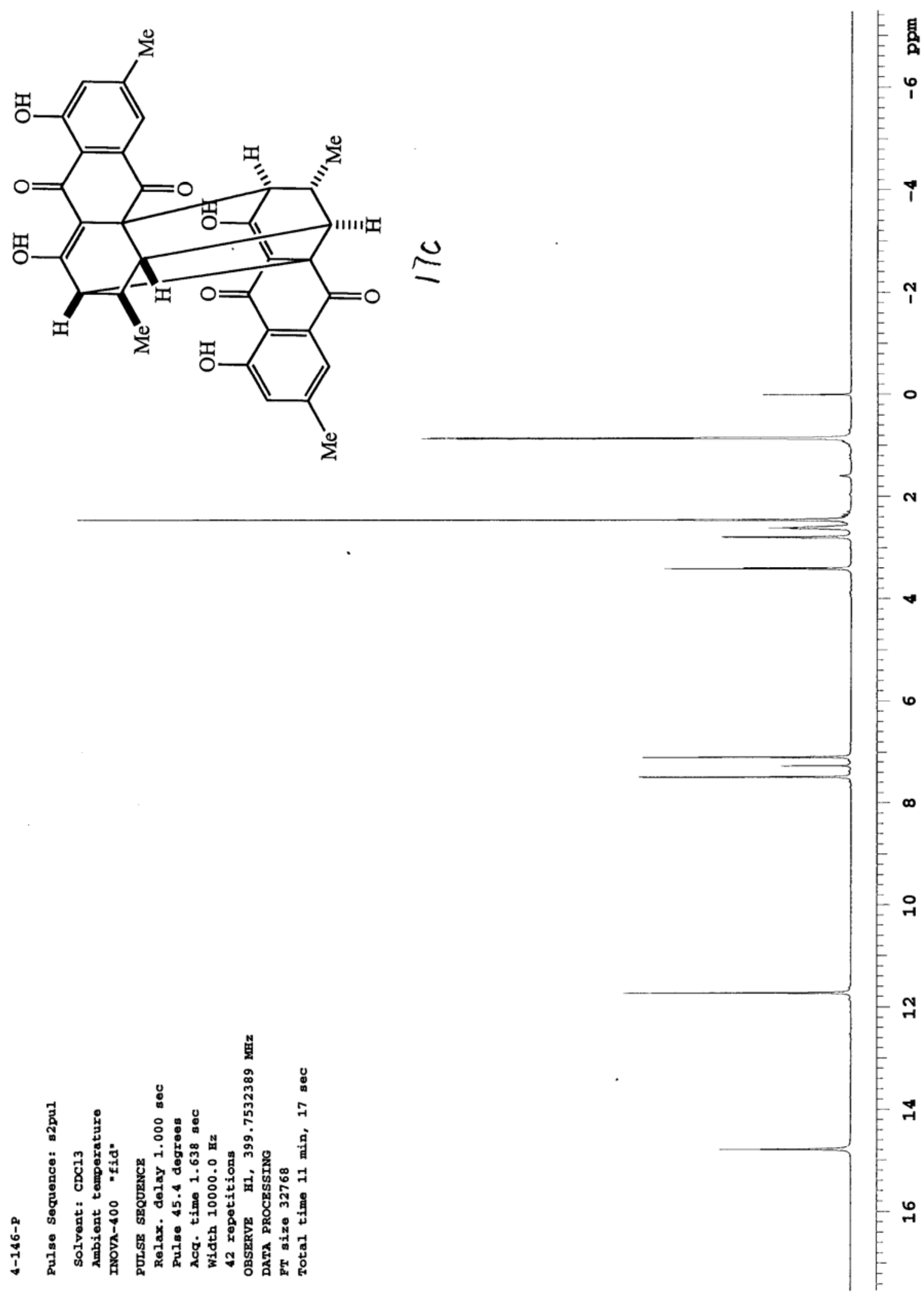

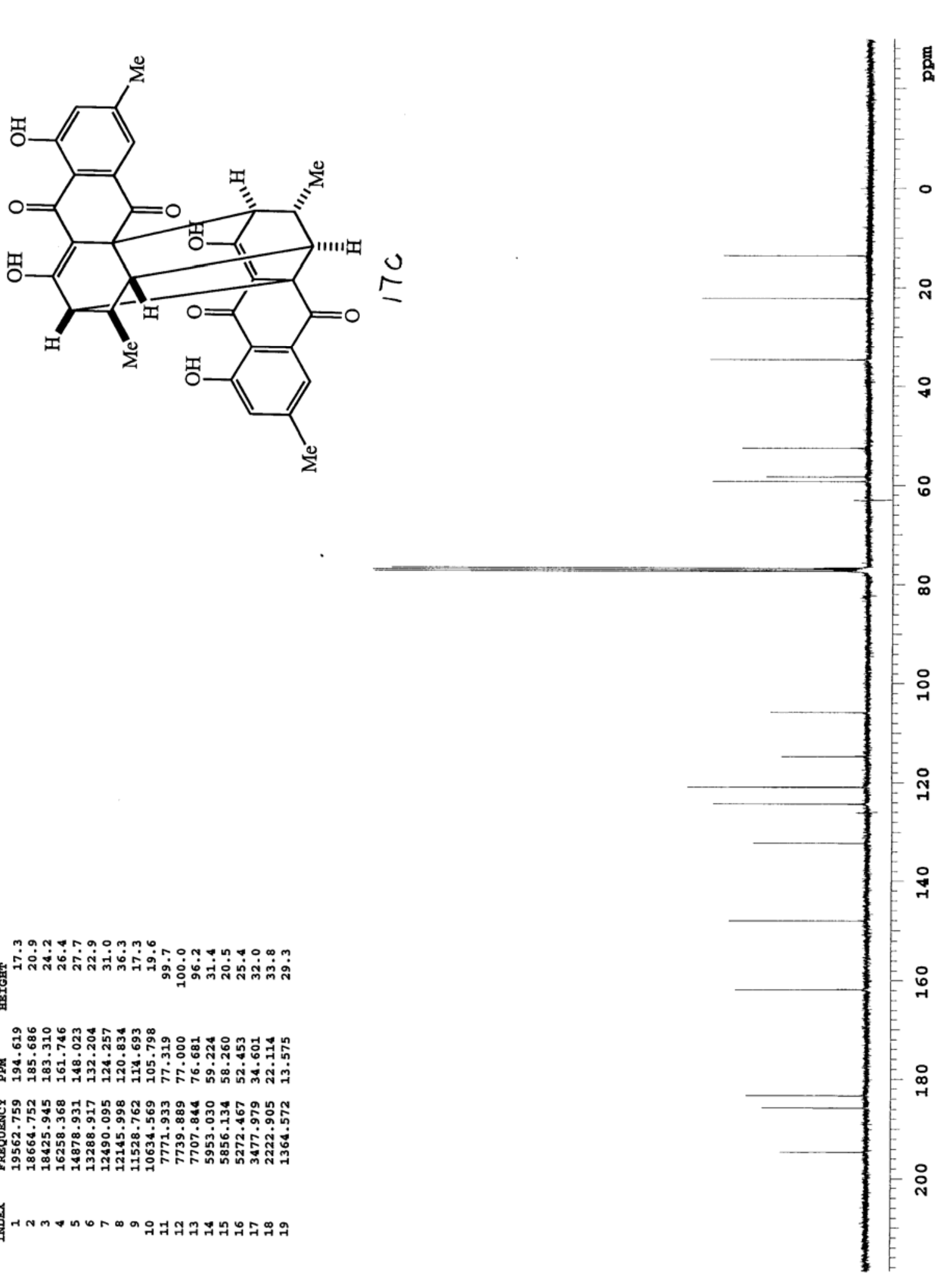

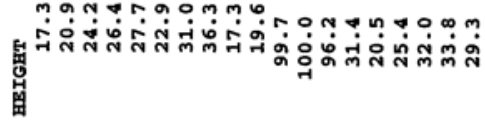

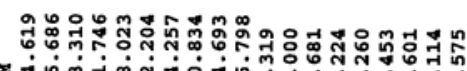

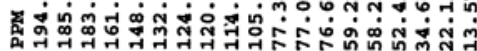

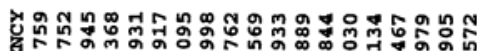

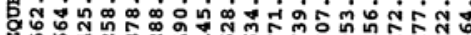

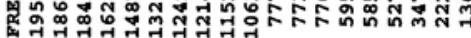

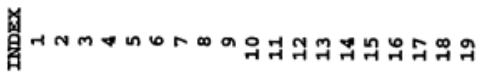

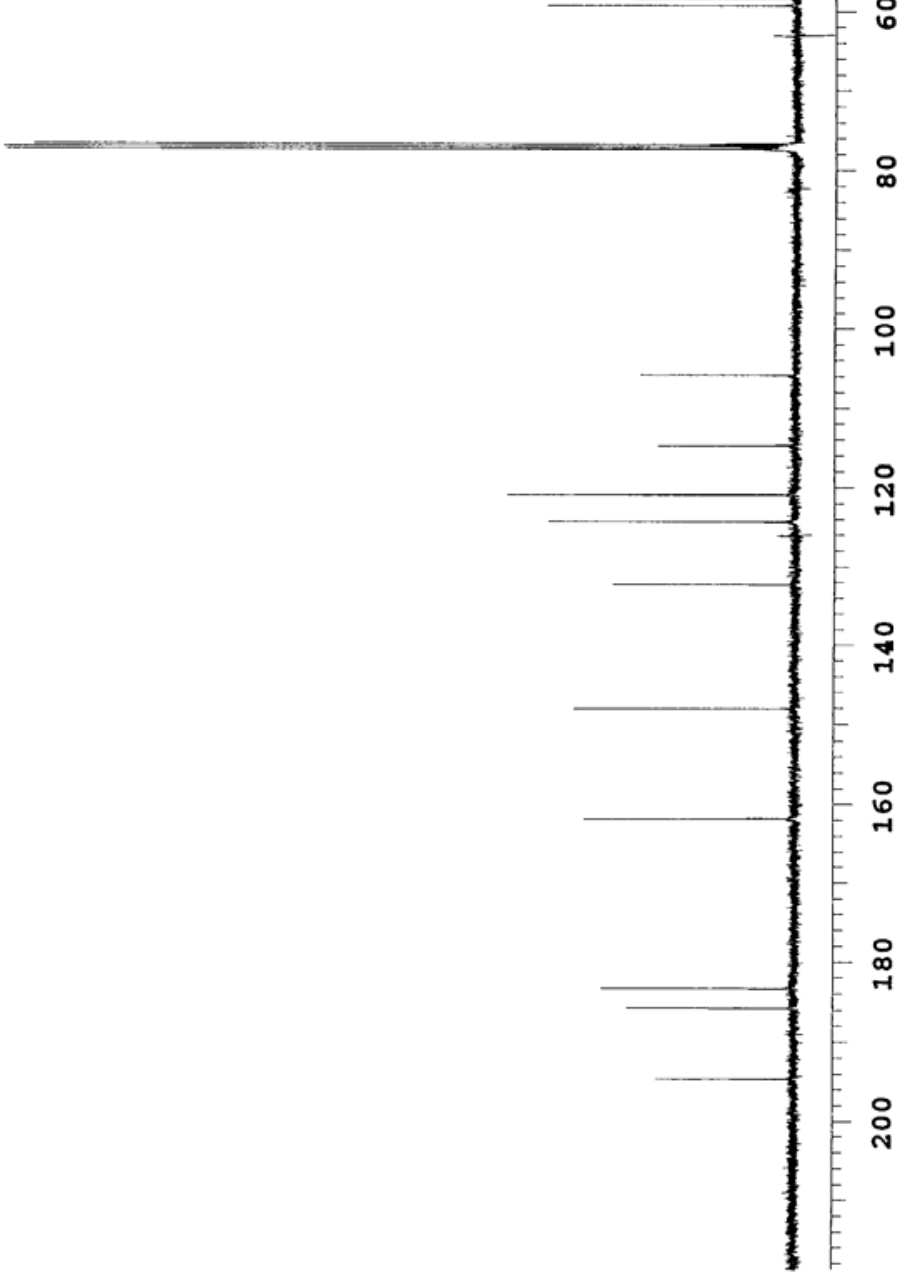



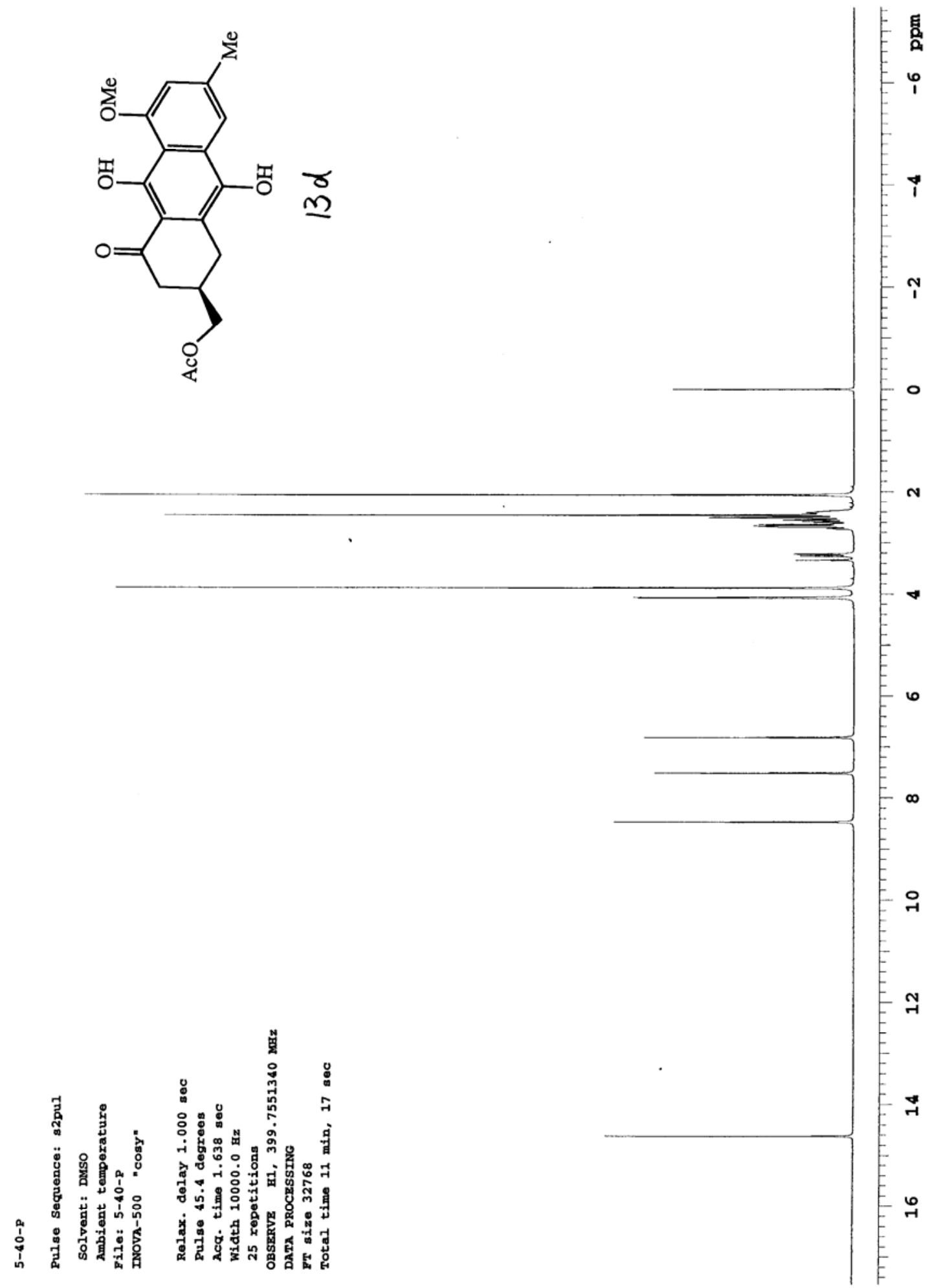

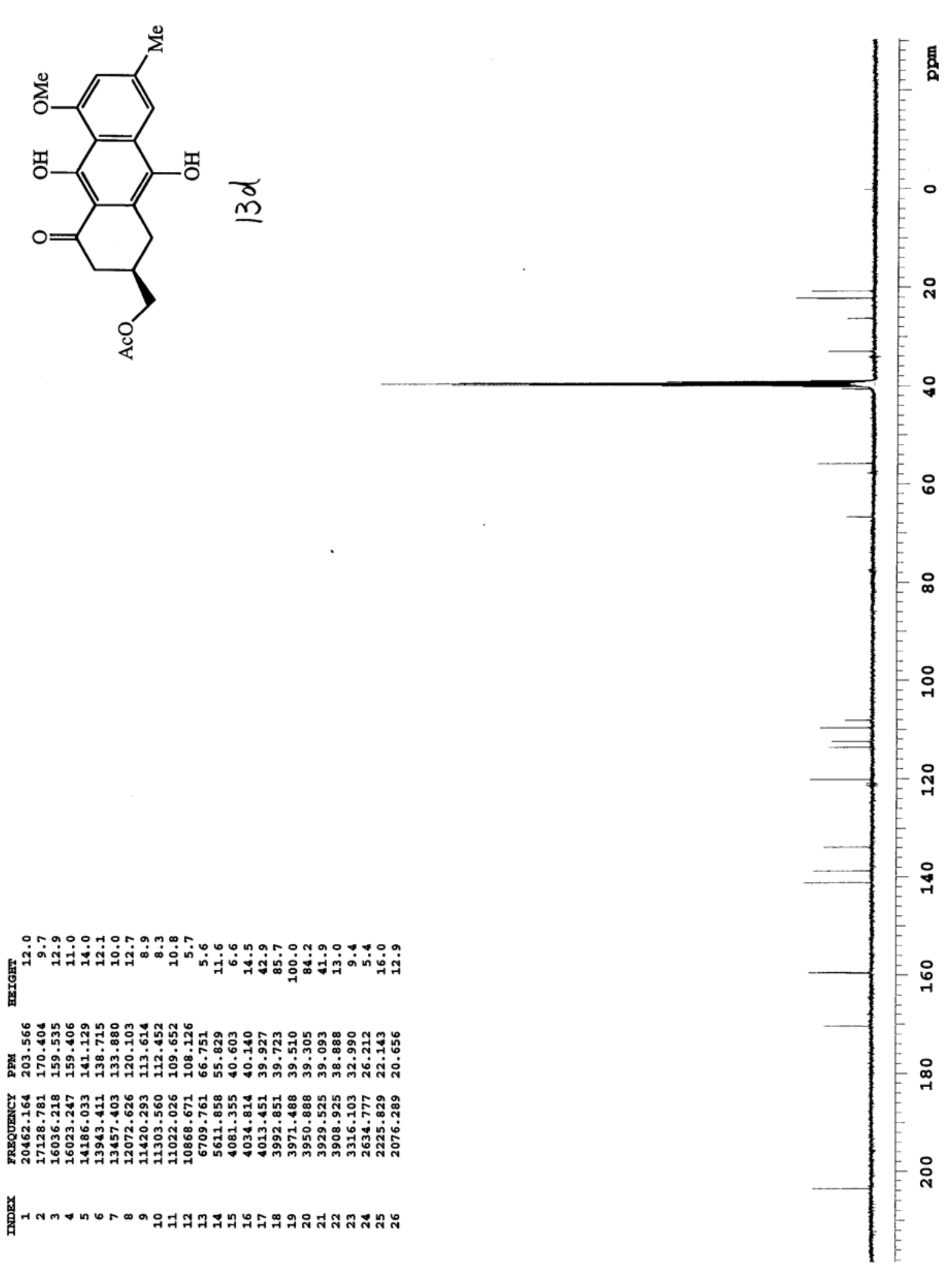

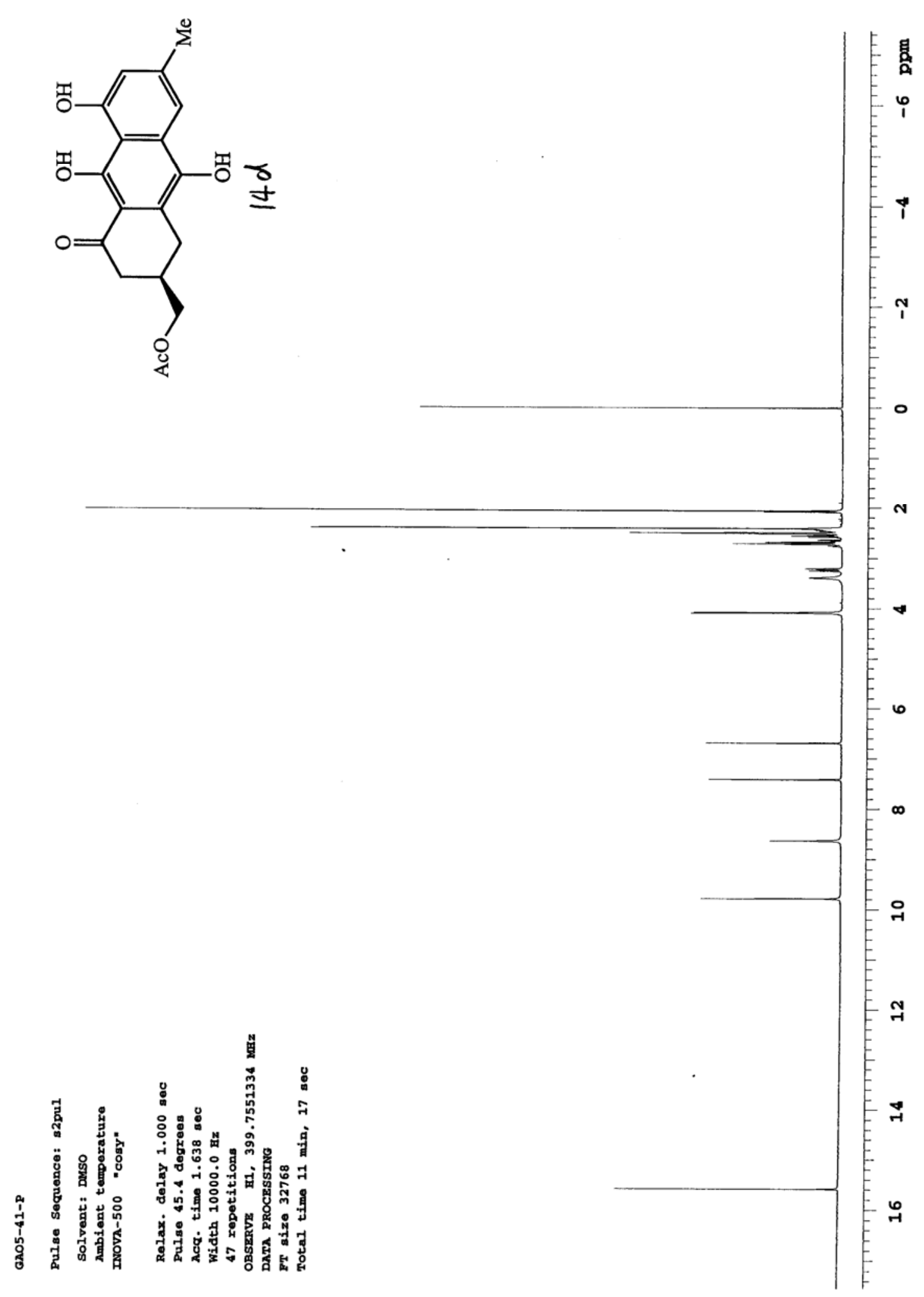


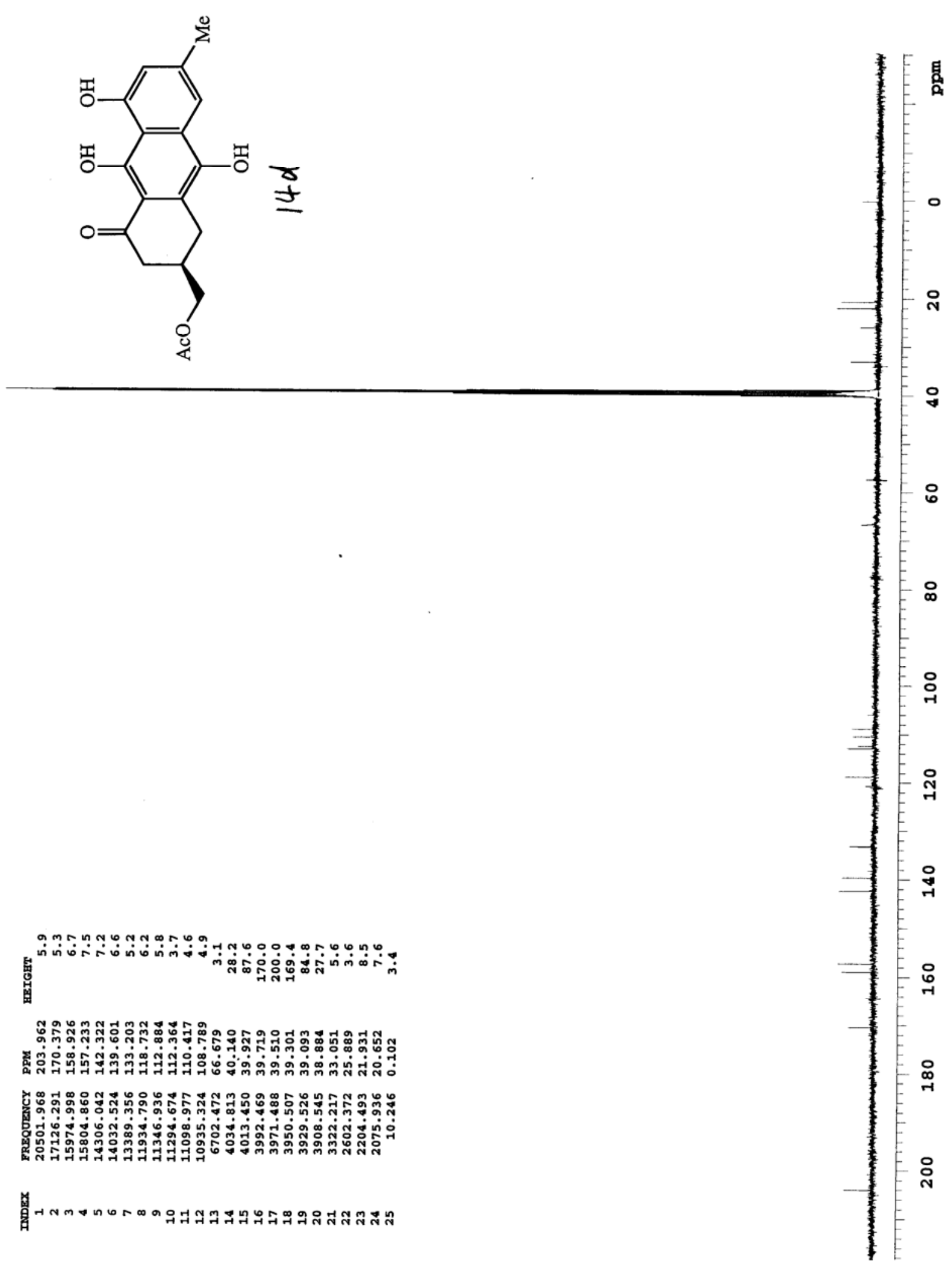




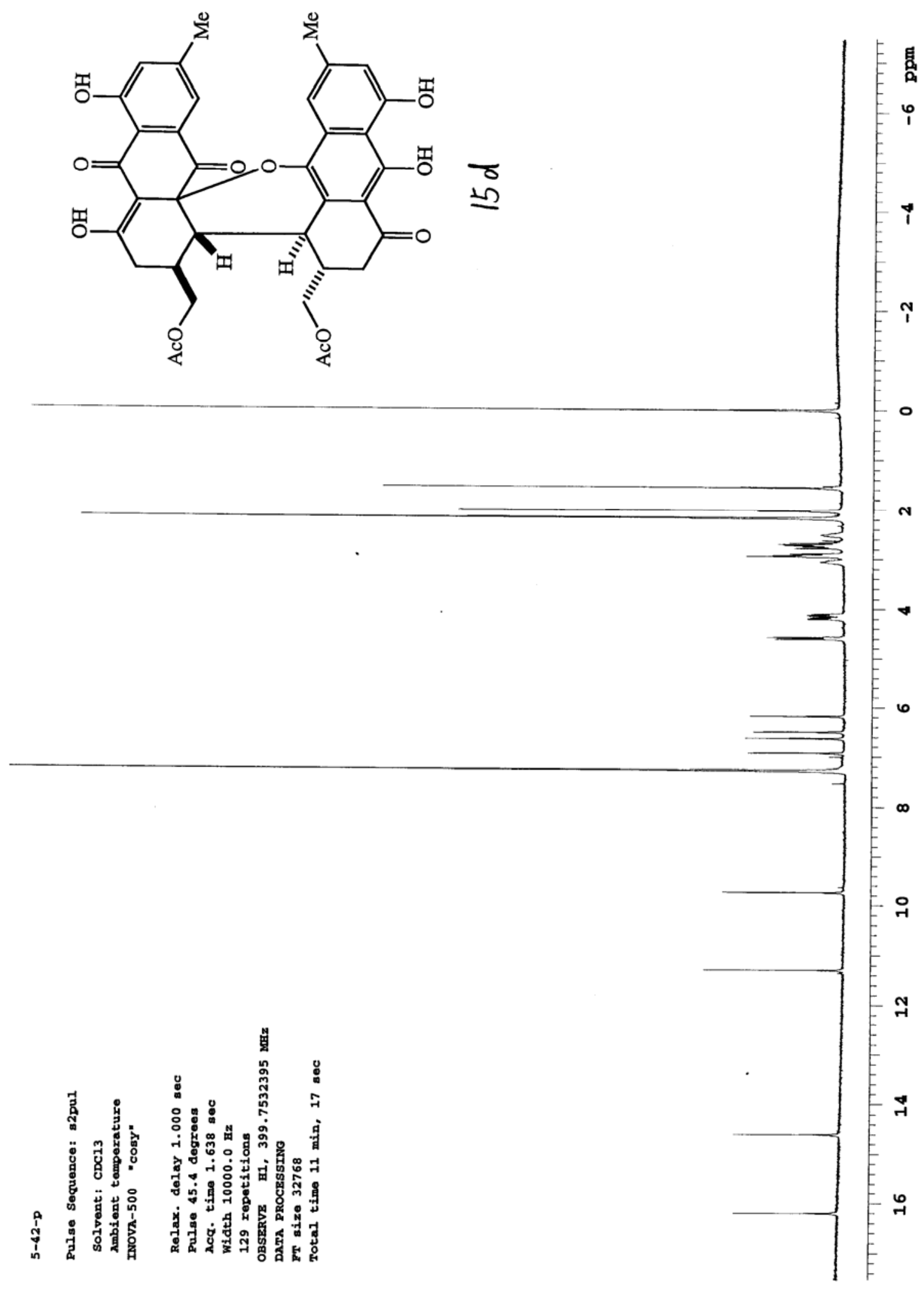




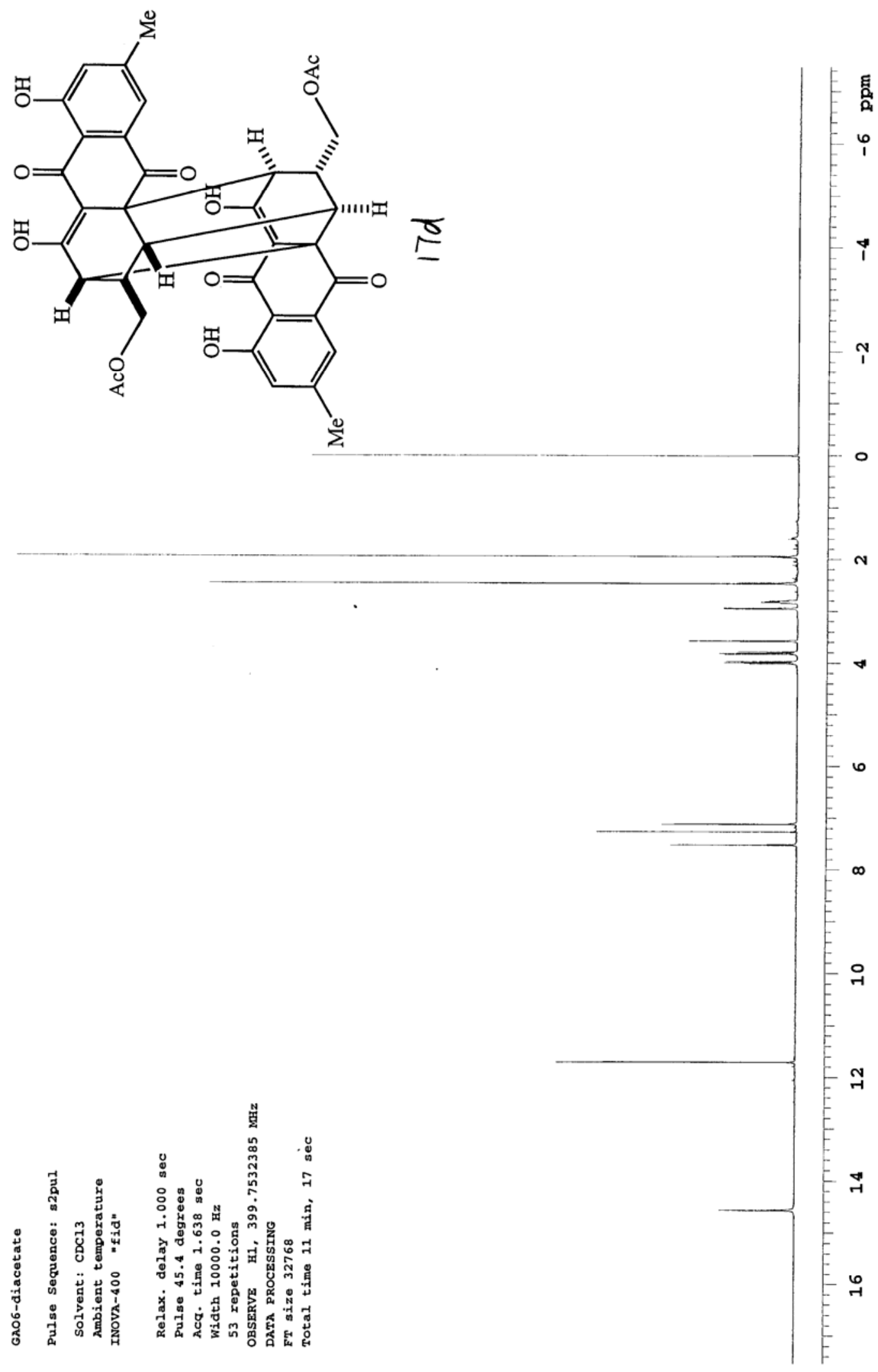



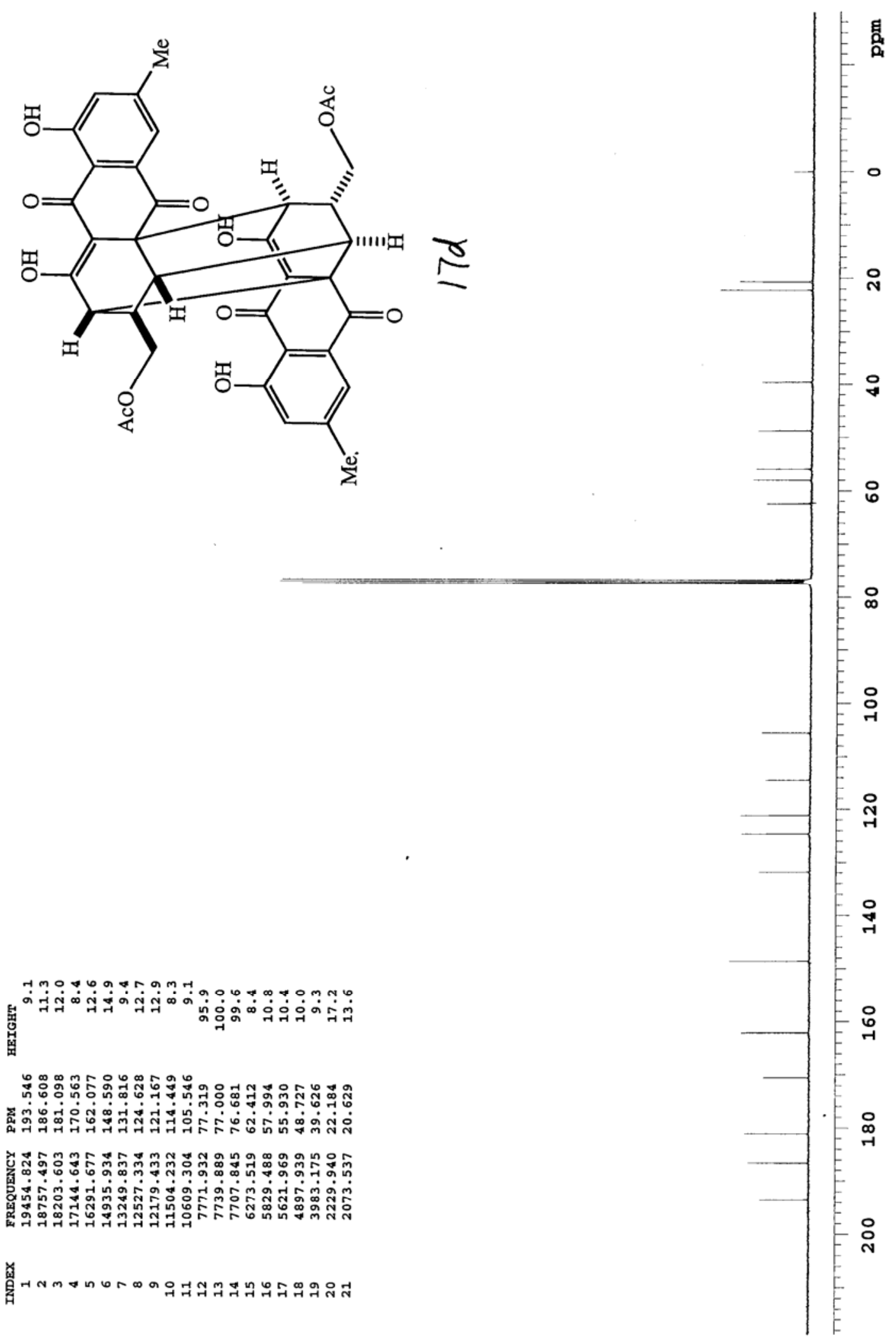


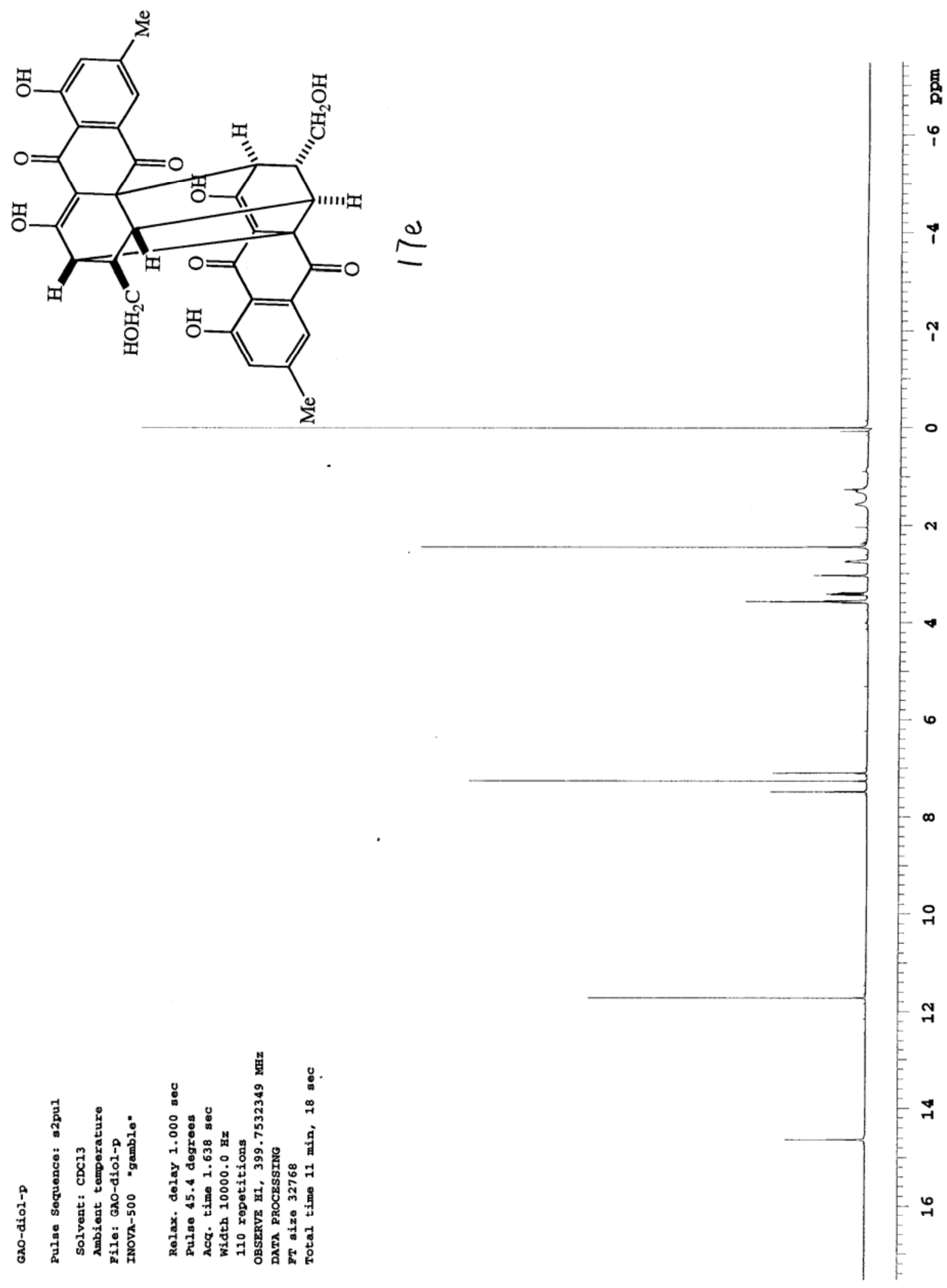



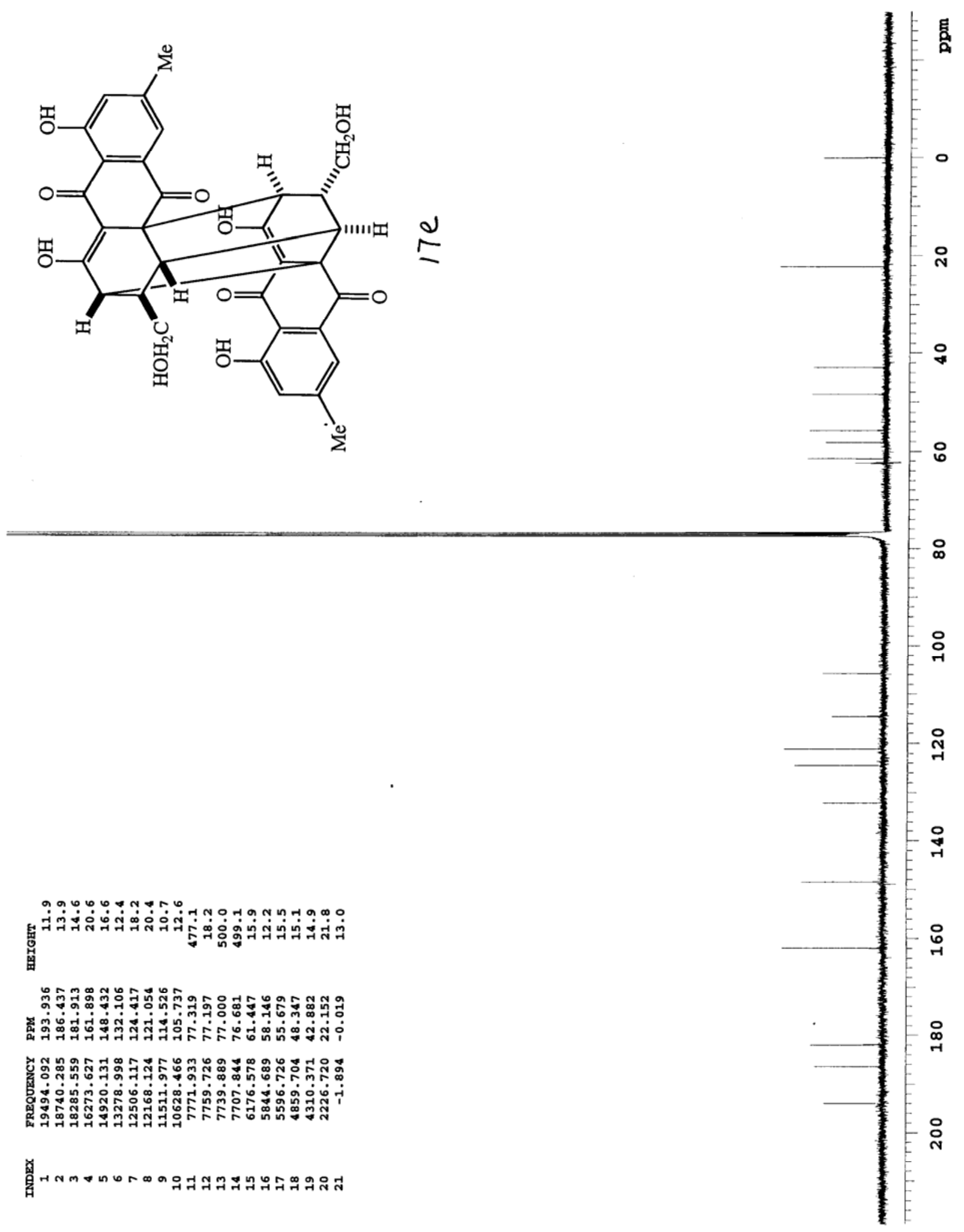


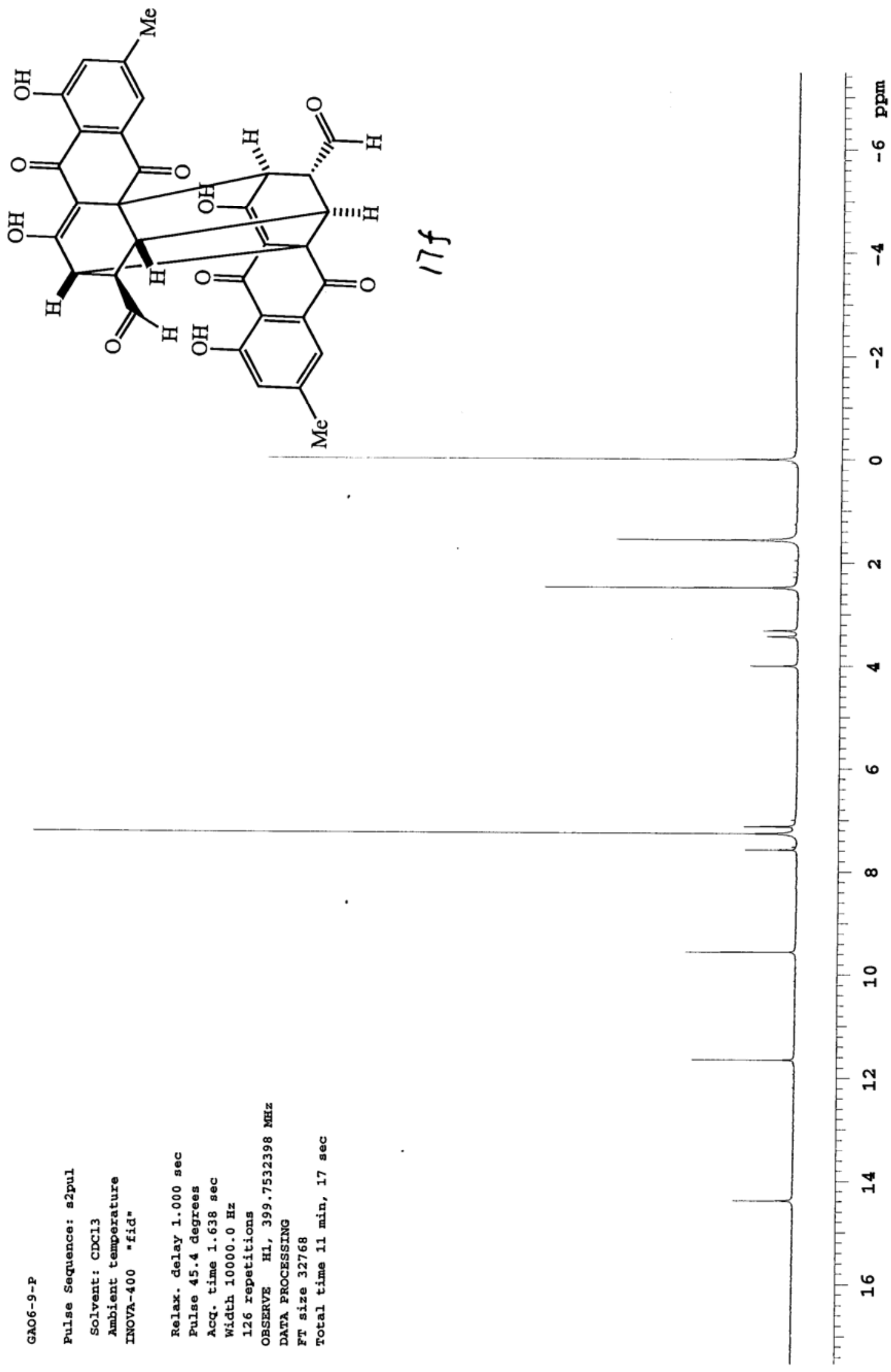




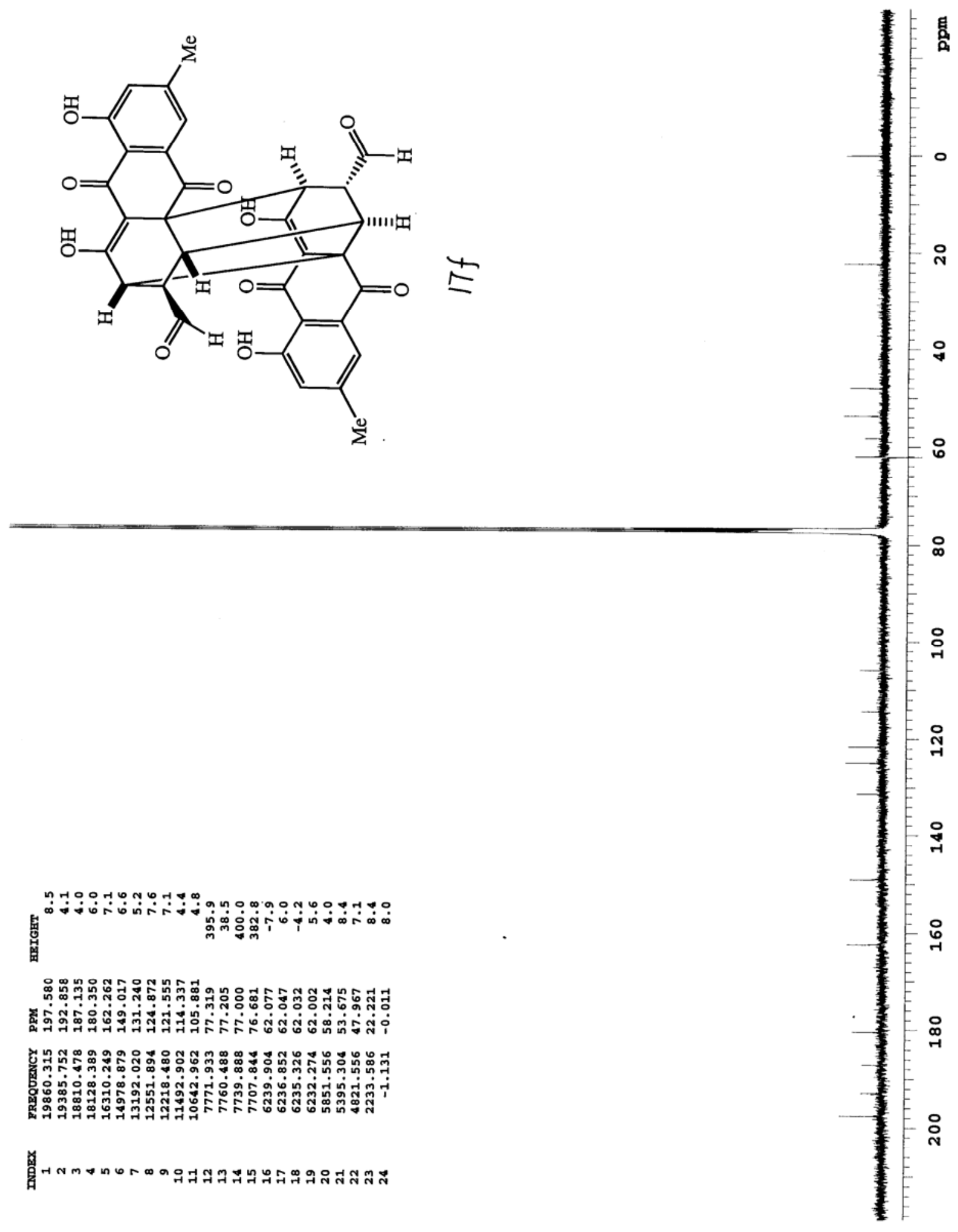




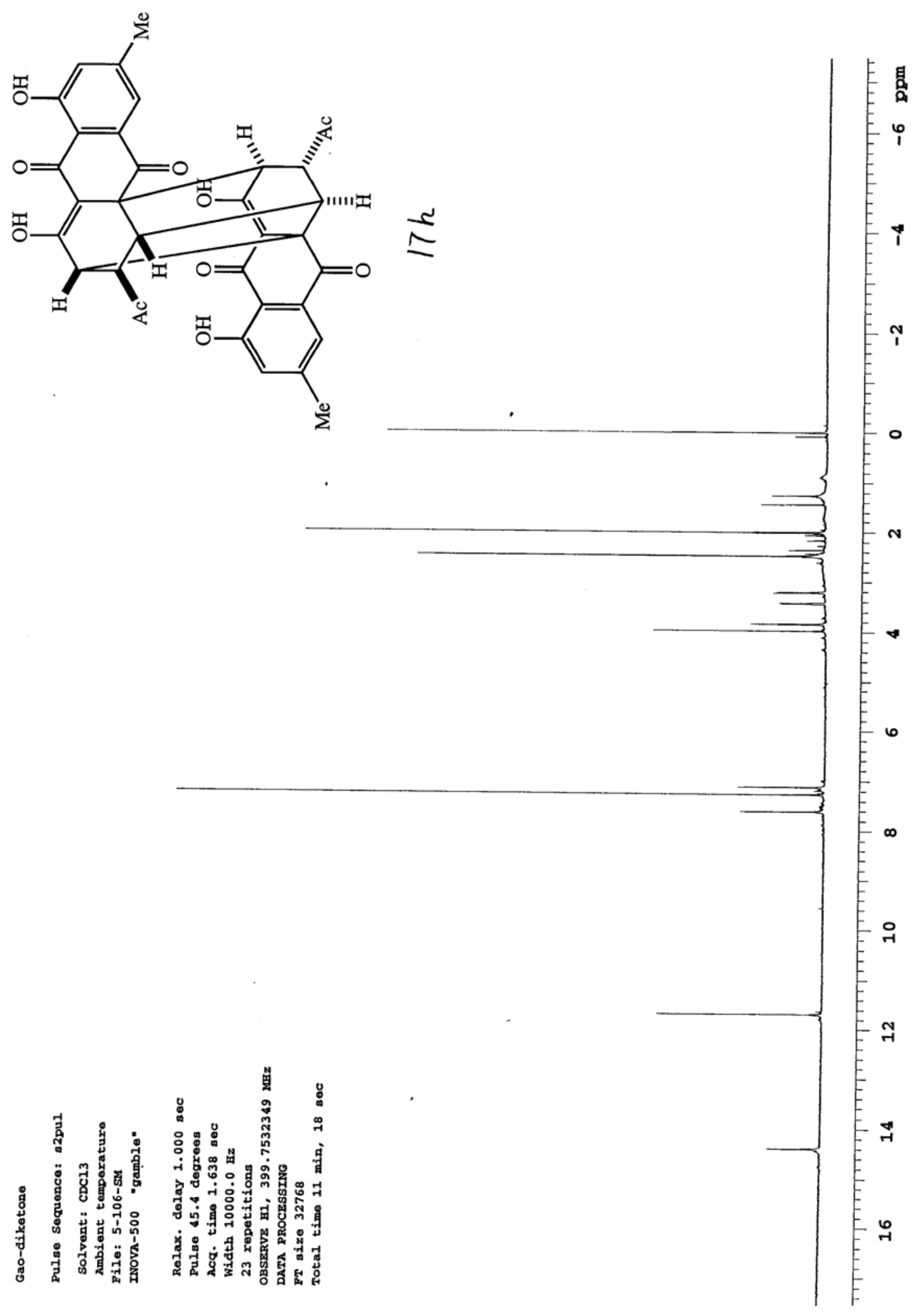




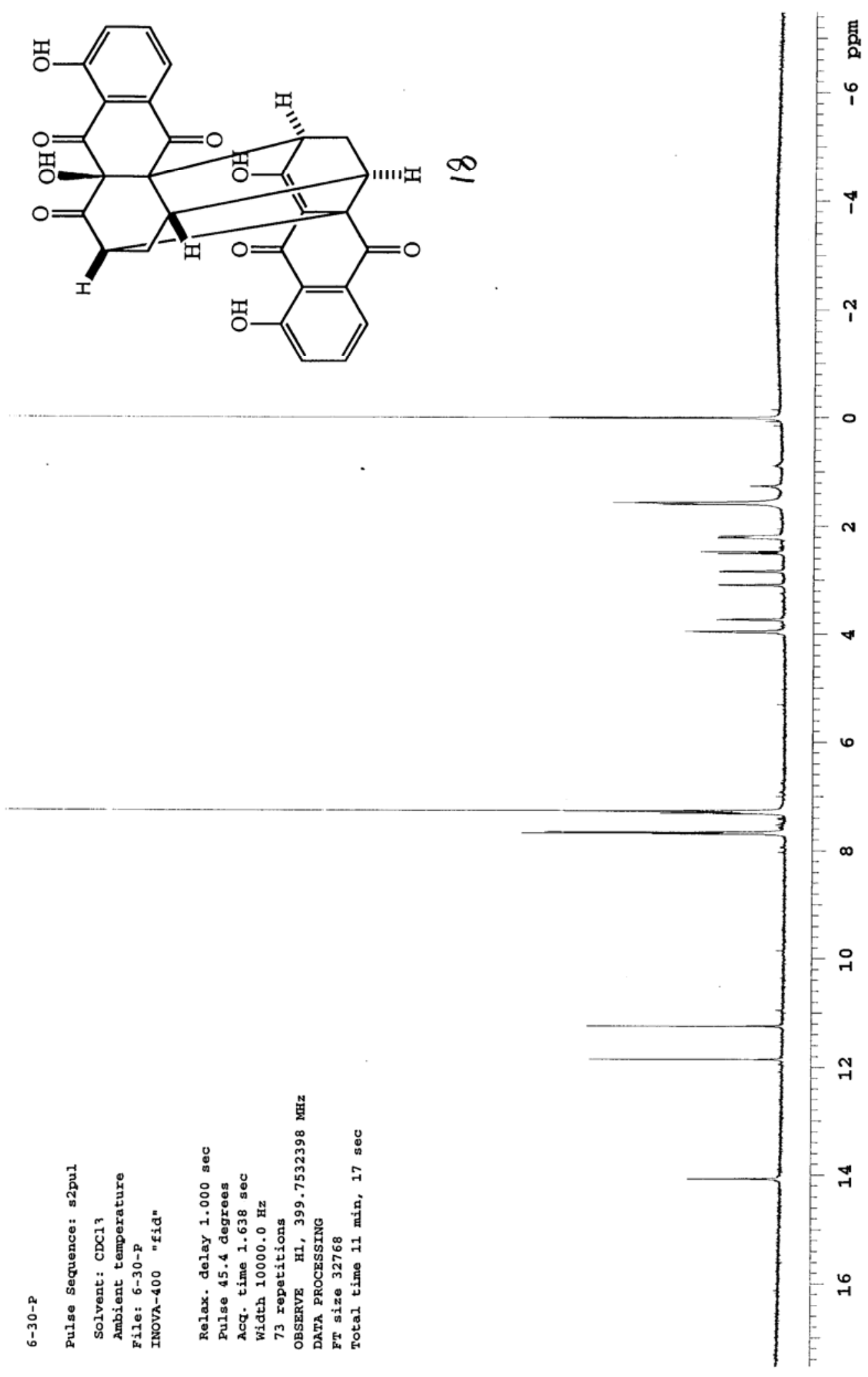




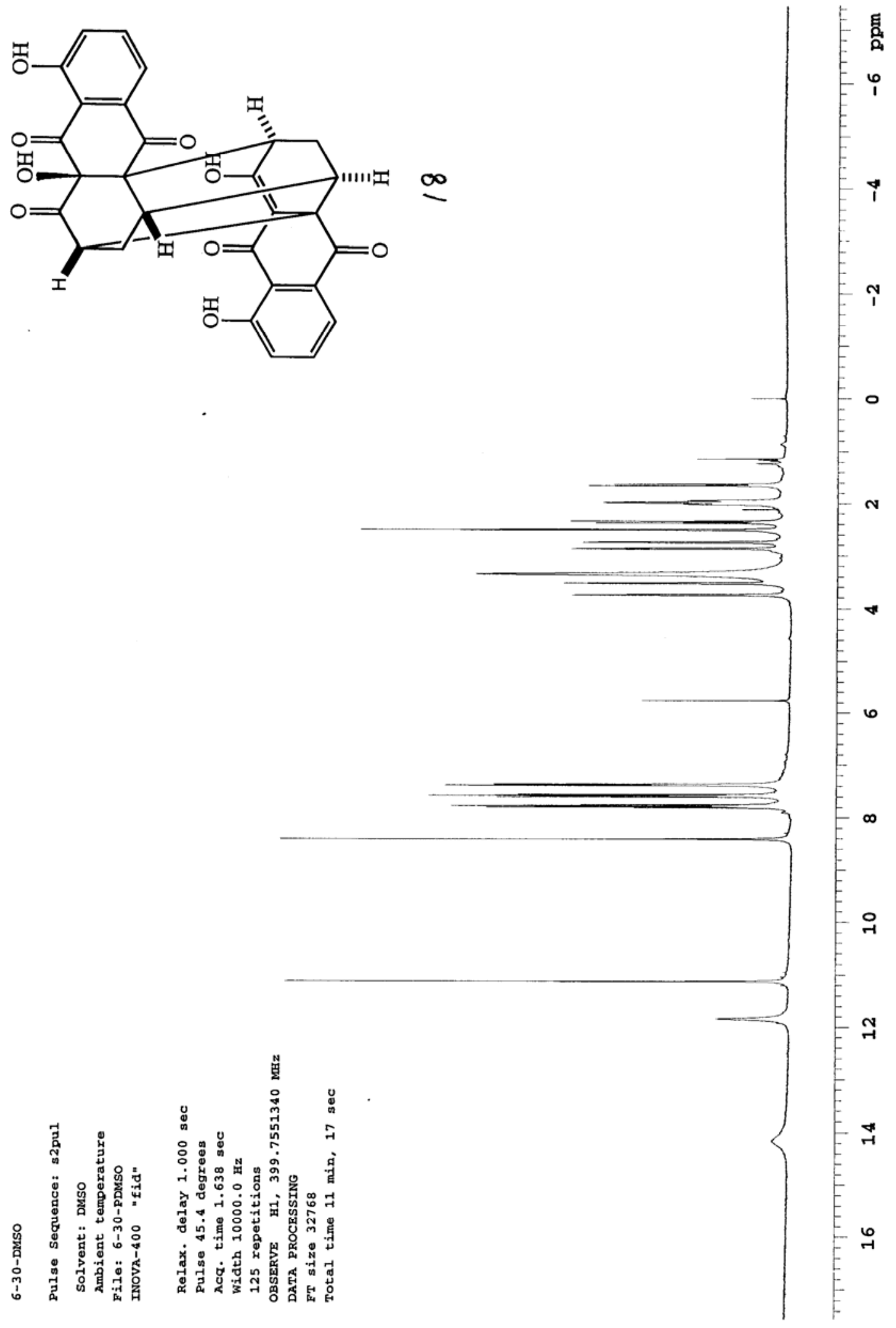




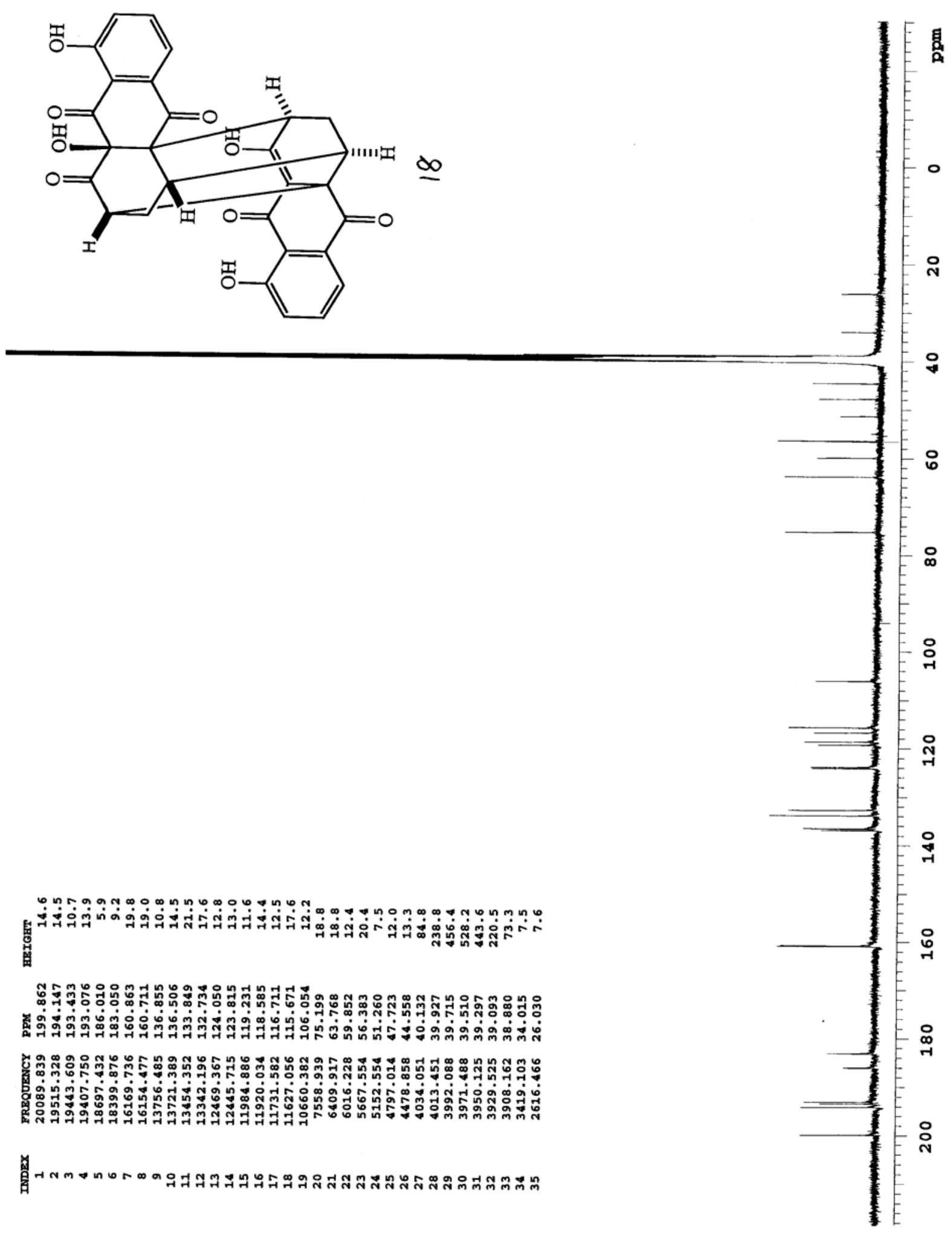




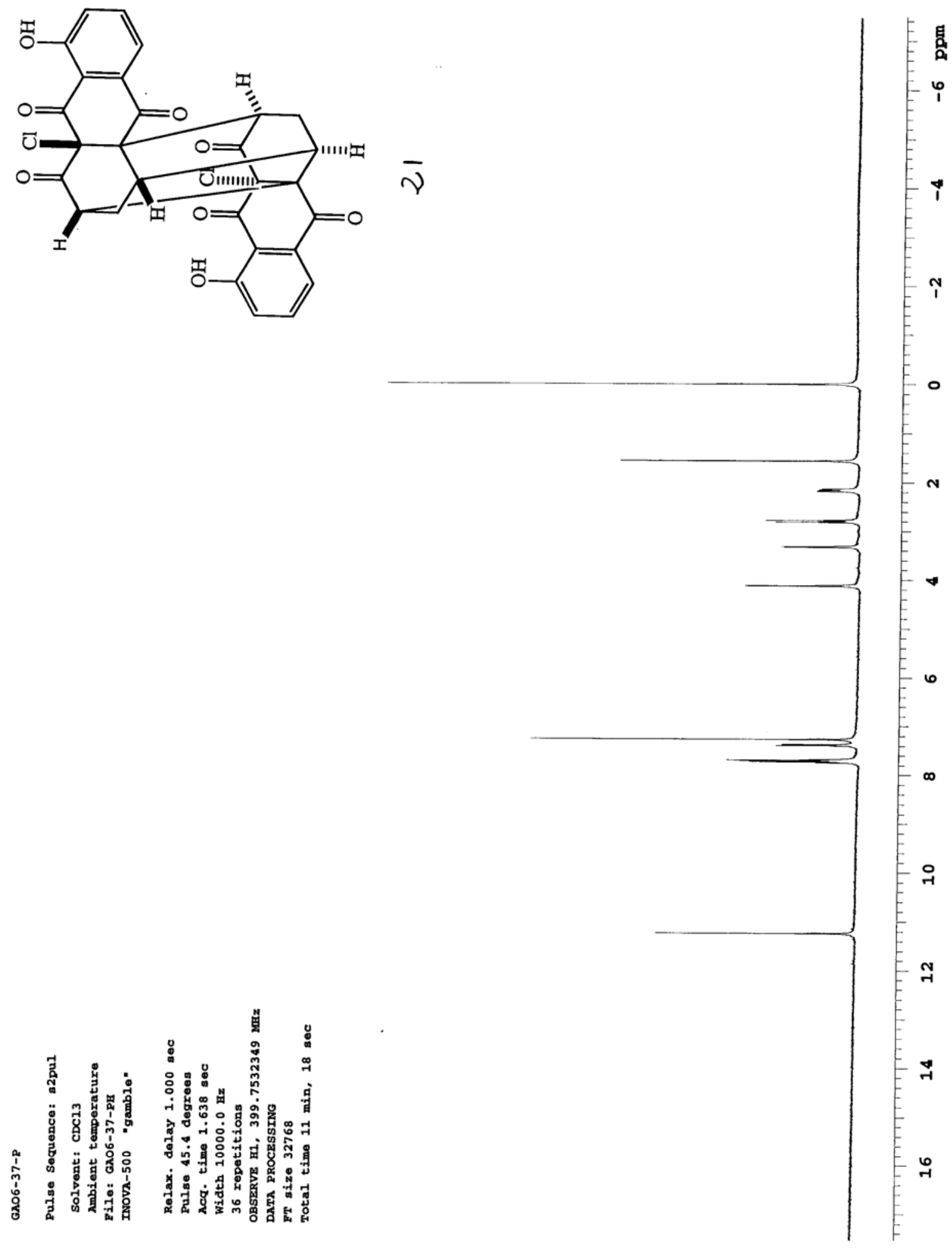



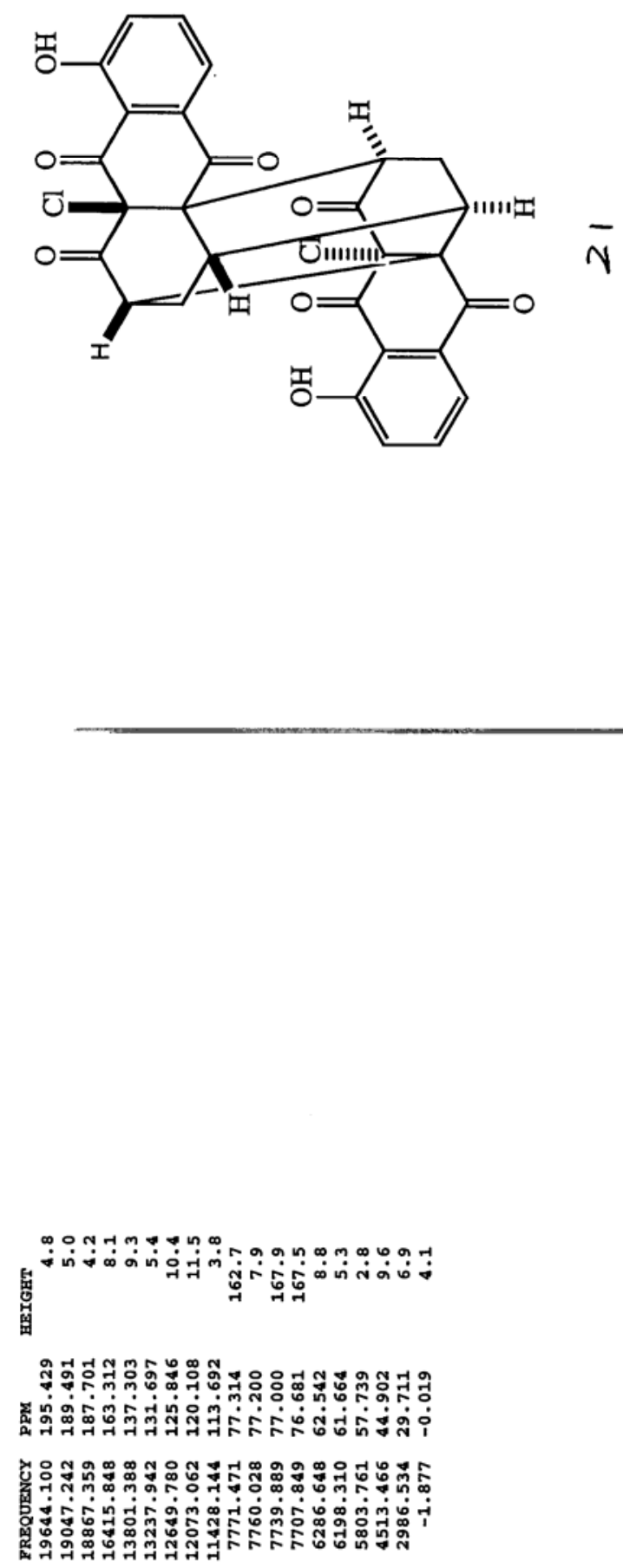

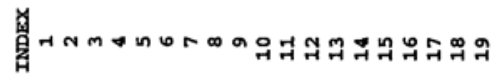

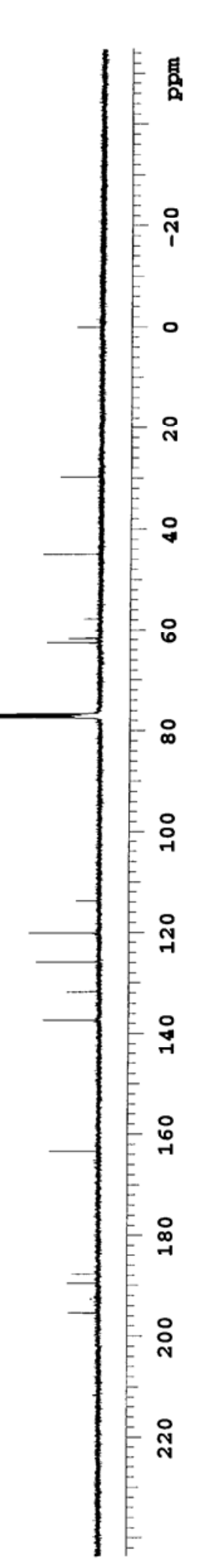




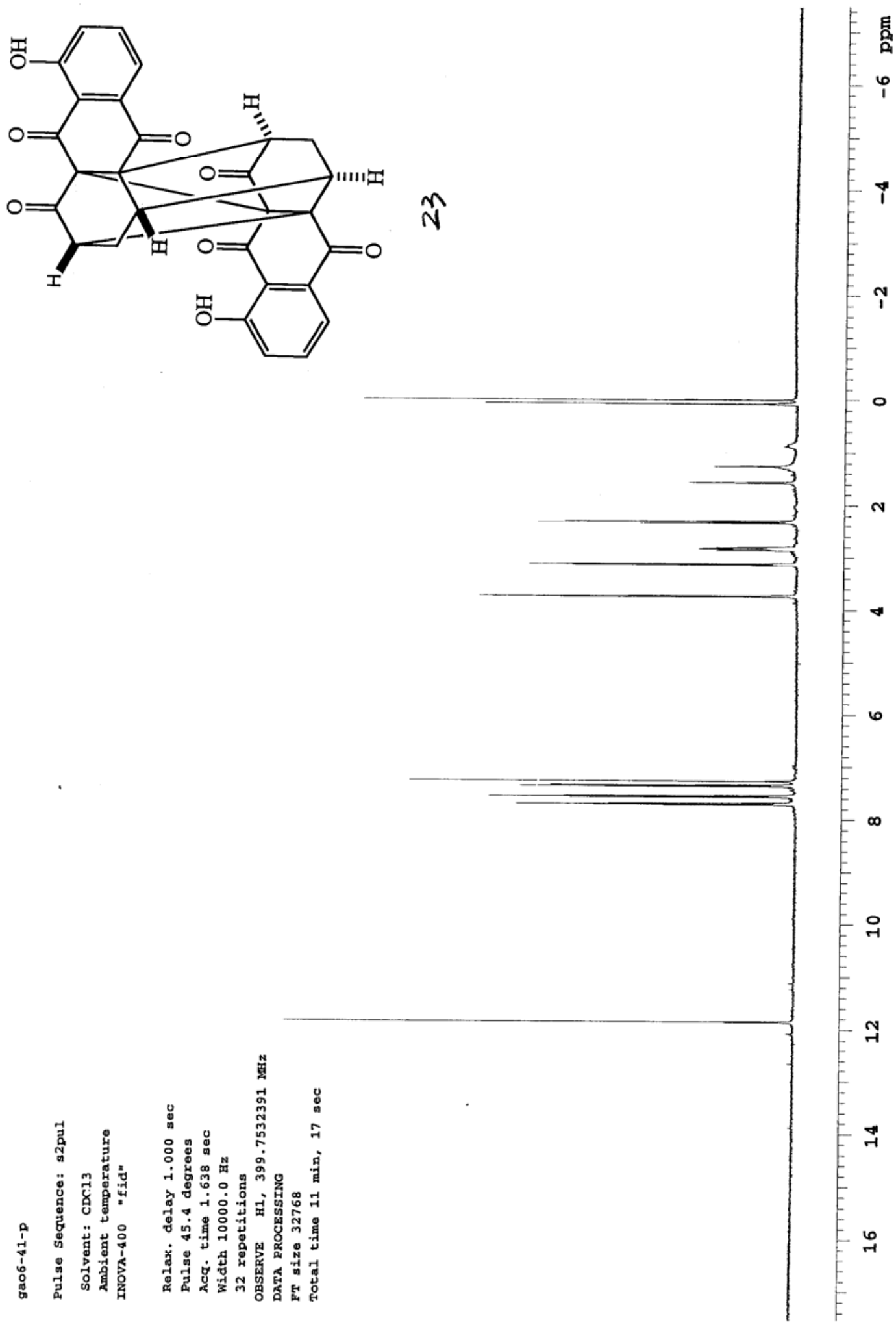




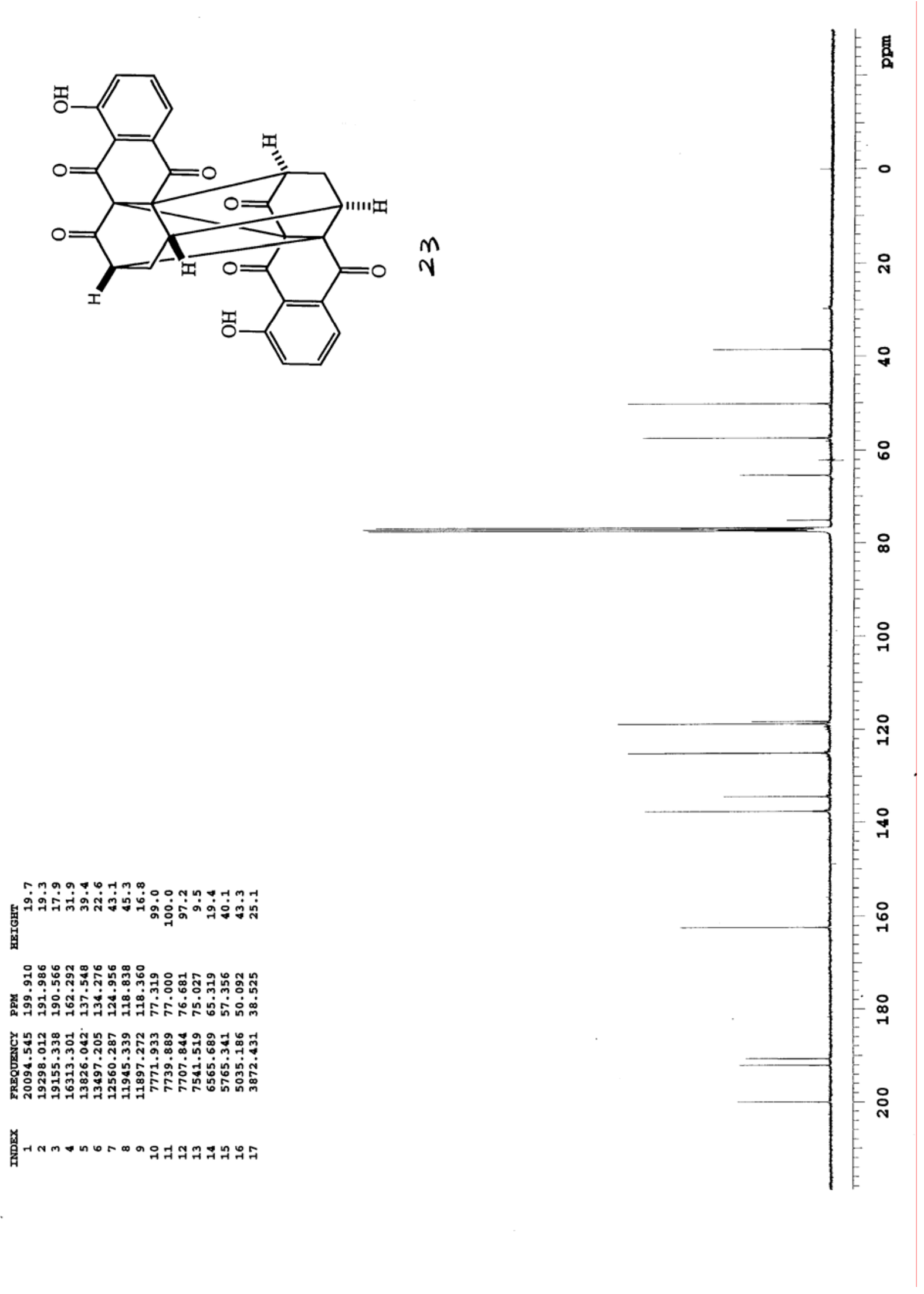

